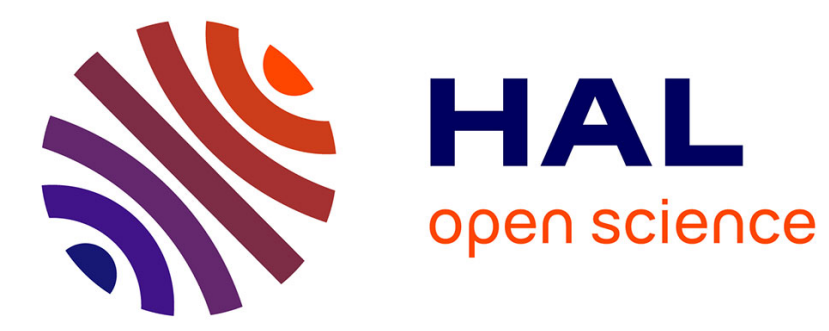

\title{
la conception du mouvement chez les anciens philosophes grecs
}

\author{
Hervé Albert-R-E Barreau
}

\section{To cite this version:}

Hervé Albert-R-E Barreau. la conception du mouvement chez les anciens philosophes grecs. 2007. halshs-00134497

\section{HAL Id: halshs-00134497 https://shs.hal.science/halshs-00134497}

Preprint submitted on 2 Mar 2007

HAL is a multi-disciplinary open access archive for the deposit and dissemination of scientific research documents, whether they are published or not. The documents may come from teaching and research institutions in France or abroad, or from public or private research centers.
L'archive ouverte pluridisciplinaire HAL, est destinée au dépôt et à la diffusion de documents scientifiques de niveau recherche, publiés ou non, émanant des établissements d'enseignement et de recherche français ou étrangers, des laboratoires publics ou privés. 


\title{
La conception du mouvement chez les anciens philosophes grecs
}

Résumé : Les anciens philosophes grecs ont proposé différentes conceptions du mouvement, dont certaines ont été rejetées par les initiateurs de la Révolution scientifique du XVII siècle, tandis que d'autres ont été accueillies et développées, en particulier le mécanisme. Dans toutes ces anciennes philosophies, à l'exception d'une seule, ce mécanisme était associé à différentes sortes d'animisme, de vitalisme ou de finalisme. Il suffit, pour s'en rendre compte, de passer en revue les différentes écoles philosophiques, depuis l'école de Milet jusqu'au néoplatonisme, en passant par le pythagorisme, l'éléatisme, les "physiologues » du temps de Socrate, Platon et Aristote, l'épicurisme et le stoïcisme. Ces écoles se répondaient les unes aux autres, en faisant la critique de leurs prédécesseurs. En relevant aujourd'hui les défauts des explications qu'elles proposent, on peut faire d'utiles rapprochements avec la science moderne, où la cosmologie et la théorie générale de l'Evolution reprennent les ambitions qui avaient été celles des penseurs grecs anciens.

\begin{abstract}
The ancient greek philosophers have offered different theories about movement, some of which have been rejected by the promoters of the seventeenth century scientific revolution, but others have been received and developed, mainly mecanicism. This mecanicism is present by all these philosophies, where it is connected, except in one, with different sorts of animism, vitalism and finalism. This can be demonstrated by reviewing the different philosophical schools, from Milet school to neo-platonism, going across pythagorism, eleatism, the physiologists contemporary with Socrates, Plato and Aristotle, epicurism and stoïcism. These schools were exercising a constant dialog and refutation between each other. While pointing out the defects inherent in their own explanations, useful rapprochements can be made to-day with modern science, where cosmology and the general theory of Evolution are taking again the ambitious goals the ancient greek philosophers persued.
\end{abstract}

Le mouvement est un phénomène si familier qu'il semble qu'il soit inutile d'avoir recours aux philosophes pour en acquérir une connaissance valide, voire une science. Pourtant, si l'on considère l'histoire de l'humanité, on constate qu'il en a été tout autrement. Ce sont les peuples qui ont réfléchi profondément sur la nature, les principes, les espèces du mouvement, qui sont parvenus à se doter de principes tels qu'ils ont rendu possible et féconde une science du mouvement. Si l'on met à part les Chinois, qui ont développé de leur côté une riche tradition, ces peuples, qui ont inventé une technique et une science du mouvement, ont tous bénéficié de l'héritage des philosophes grecs. Même si, à partir de la Renaissance en Europe, ces peuples ont récusé l'héritage de la philosophie d'Aristote, qui s'était imposée durant tout le Moyen-Age musulman et chrétien, et se sont réclamés, à tort ou à raison, d'autres auteurs grecs, antérieurs ou postérieurs à Aristote, c'est toujours de l'un ou de l'autre de ces philosophes qu'ils se sont réclamés pour doter leur inspiration de quelque autorité. Nos auteurs modernes et contemporains ont trouvé, en effet, dans le riche héritage de la Grèce, une si abondante palette de doctrines, apparentées les unes aux autres, et critiques à l'égard de celles qui les avaient précédées, qu'ils n'ont eu, le plus souvent, qu'à faire un choix, en sachant les avantages et les inconvénients qui pouvaient en résulter. Il est indéniable que les philosophes grecs ont préparé la science occidentale du mouvement, qui s'est propagée dans le monde entier, et une sagesse à l'égard du mouvement qui n'est pas inférieure à d'autres traditions plus anciennes que celle dont ils se réclamaient. On se contentera d'évoquer ici les 
différentes étapes de cette préparation, en les rattachant aux contextes de la réflexion physique ou morale où elles ont pris leur sens originaire, avant de s'offrir à la réflexion ultérieure des penseurs qui y ont eu recours. Ce faisant, on s'efforcera d'éviter tout anachronisme, comme cela a été la préoccupation majeure des spécialistes récents auxquels on ne manquera pas de faire appel dans les cas nombreux où l'interprétation des textes disponibles est loin d'être obvie.

\section{1. le mouvement dans l'Ecole de Milet}

Trois noms se détachent dans la colonie grecque d'Asie mineure qui se développa, au $\mathrm{VII}^{\mathrm{e}}$ et au $\mathrm{VI}^{\mathrm{e}}$ siècle avant notre ère, à Milet, la plus florissante sans doute, à cette époque, des cités grecques qui vivaient des échanges commerciaux. Thalès, Anaximandre et Anaximène, dont seuls les deux derniers ont écrit chacun, selon la tradition, un ouvrage De la Nature, bien qu'ils ne soient connus que par des allusions ou des esquisses historiques d'auteurs très postérieurs, témoignent assurément d'un esprit tout différent de celui qui inspire les mythologies primitives; c'est chez eux la recherche manifeste d'une explication rationnelle des mouvements les plus frappants, qui leur a valu de figurer comme les pères de la philosophie et de la science grecques.

a) On sait que ce qui rendit célèbre Thalès fut la prédiction exacte d'une éclipse totale du Soleil. C'est donc que les mouvements astronomiques retinrent dès l'origine l'attention des penseurs milésiens. Mais les mouvements généraux qui se passent sur Terre attirèrent également la curiosité de Thalès, puisqu'il proposa d'y voir les transformations que subit l'eau, considéré comme l'élément primordial. Il suffit de considérer la course des nuages, en effet, d'y voir l'origine des orages et des pluies, qui donnent naissance aux fleuves, qui se jettent dans la mer, pour imaginer que la Terre flotte sur la mer, qui serait ainsi l'origine de tout ce qui sort d'elle par évaporation, même du feu des orages. Selon le témoignage d'Aristote (De l'Ame, 405 a 19,411 a 7), Thalès aurait dit que « l'âme est motrice, s'il est vrai qu'il disait de la pierre magnétique qu'elle a une âme parce qu'elle meut le fer». Le magnétisme lui révélait ainsi qu'il y a des mouvements non mécaniques, ceux qui ne sont pas dus à la pression, mais à une certaine attirance. Serait-ce de l'animisme ? Il est difficile de se faire une idée précise de cet animisme non mythologique, qui se basait sur des faits bien observés, sur lesquels la postérité devait réfléchir à son tour. En tout cas, Aristote pouvait rapprocher une telle conception animiste d'une autre affirmation, transmise par la tradition, selon laquelle, d'après Thalès, « tout est plein de dieux ».

b) Anaximandre, qui fut sans doute, l'élève de Thalès, a présenté une cosmologie très différente de celle de son maître et où l'explication mécanique a une plus grande place. La Terre n'est plus portée par l'eau, mais est suspendue au milieu du Ciel, comme une colonne dont nous occupons la section supérieure (qui est donc plate). A égale distance de cette colonne, tournent des cercles de feu, qui sont enveloppés d'une gaine percée de trous, que nous identifions comme des astres. Nous avons donc affaire à un véritable modèle, qui explique les éclipses et les phases de la Lune. Les unes et les autres seraient dues à la disparition de ces trous, dont la cause serait soit une obturation solide ou partielle de ces orifices, soit la rotation sur elle-même de la roue, qui ne les présenterait pas toujours tournés vers nous. Nous verrons que l'explication du mouvement apparent des astres par des cercles dont le centre est la Terre aura une longue postérité. Mais, ce qui est frappant chez Anaximandre, c'est que l'explication scientifique est poussée jusqu'à un plan que nous considérons comme métaphysique, puisque la suspension de la Terre est assurée par l'Infini 
qui l'entoure, sans lui imposer mouvement ni orientation. C'est l'Infini, non l'eau, qui est donc,pour Anaximandre, le premier principe, inengendré et impérissable, qui contient et dirige toutes choses. En s'appuyant sur des expressions attribuées à Anaximandre, Léon Robin a pu écrire : " Il (l'Infini) est ce dont sortent toutes choses et en quoi elles reviennent selon le mouvement éternel, qui paraît bien être, à considérer les oppositions et les rapprochements établis à ce propos par Aristote, un processus de séparation et de réunion des contraires, dont la forme originelle serait un tourbillonnement chaotique ${ }^{1}$. Anaximandre est donc un penseur de l'Evolution cosmique. A partir du chaos, se distinguent le Chaud et le Froid. Le Chaud forme la sphère de flamme (et d'air), qui se rompt en produisant les cercles dont nous voyons les trous. La Terre serait antérieure aux astres, puisqu'elle appartiendrait au Froid, tout comme l'eau. Or l'Evolution se poursuit sur Terre. Les premiers vivants se sont formés dans un mélange de terre, d'air et d'eau. A l'origine, ils étaient tous des poissons. L'homme s'est trouvé longtemps porté par de tels êtres vivants, avant de pouvoir subsister par lui-même. Vision grandiose, qui s'accompagne de l'hypothèse d'univers multiples. Car de l'Infini peuvent naître des mondes multiples qui n'ont chacun qu'une existence bornée, puisque le Chaud et le Froid s'anéantissent finalement l'un l'autre. Selon un texte conservé par Simplicius $\left(\mathrm{VI}^{\mathrm{e}}\right.$ siècle de notre ère), le mécanisme des transformations serait dominé par une loi de justice qui fixerait l'expansion de chaque contraire : " $\mathrm{Ce}$ dont la génération procède pour les choses qui sont, est aussi ce vers quoi elles retournent sous l'effet de la corruption, selon la nécessité ; car elles se rendent mutuellement justice et réparent leurs injustices selon l'ordre du tempsa $»^{2}$. Tout vient de l'Infini et y retourne, chassé de l'existence par son contraire.

c) Anaximène succède à Anaximandre dans l'Ecole de Milet. Il intègre à la fois l'enseignement de Thalès et d'Anaximandre, puisqu'il déclare qu'il n'y a qu'un seul principe, qui n'est pas pour lui l'eau mais l'air, et que cet air est infini. Il reprend aussi l'animisme de ses prédécesseurs, puisque l'âme pour lui est de l'air qui fait l'unité du corps. Tout changement est dû à des condensations ou des raréfactions de l'air, selon que ce dernier se contracte ou se relâche. A la condensation de l'air, Anaximène rattache le Froid; à la raréfaction, le Chaud. Le feu est donc de l'air raréfié ; la condensation de l'air produit, au contraire, l'eau, puis la terre. Tous les milésiens ont donc cherché, à partir de l'observation de mêmes phénomènes, à trouver la substance qui demeurait la même, à travers la variation de ses apparitions. Tous ont également montré que ces variations sont, d'un côté, des phénomènes mécaniques, mais, d'un autre côté, des manifestations d'un animisme, puisque l'air est, pour Anaximène, une puissance vivante, un dieu. La cosmologie d'Anaximène diffère un peu de celle de ses prédécesseurs, puisque la Terre repose sur l'air dont elle contraint la masse à rester compacte et immobile. La raréfaction croissante des vapeurs produit les astres. Ces derniers sont en rotation par rapport à la Terre, et, parmi eux, Anaximène distingue les étoiles fixes, qui gardent toujours leur distance, et qui sont comme des clous attachés à une surface cristalline. Pour cette raison les historiens ont pu voir dans Anaximène l'initiateur de l'astronomie grecque.

\footnotetext{
${ }^{1}$ L.ROBIN, La pensée grecque et les origines de l'esprit scientifique, La Renaissance du Livre, 1923, réédité par Albin Michel, 1963 et 1973,p.63

${ }^{2}$ SIMPLICIUS, Physique, 24, 13, cité par J.P.Dumont,D.Delattre,J.L.Poirier, Les

Présocratiques, Gallimard, Edition de la Pléiade, p.39,frag.1
} 


\section{2. le pythagorisme et l'éléatisme}

On sait qu'à partir de 546 (avant notre ère), l'Ionie est soumise par les Perses, et que la grande ville de Milet fut ruinée en 494. La disparition de l'Ecole de Milet est donc attribuable à ces événements. Mais la cosmologie ionienne fut reprise, non plus à l'Est mais à l'Ouest de la Grèce, dans les colonies d'Italie du Sud et de Sicile, qui furent souvent en rivalité avec les cités de l'Attique et du Péloponnèse. On doit à l'activité intellectuelle qui se développa dans cette Grande Grèce l'éclosion de deux écoles importantes : le pythagorisme et l'éléatisme

a) Selon le pythagorisme, les nombres sont l'essence de la réalité, que celle-ci soit matérielle ou spirituelle. Le point c'est 1 , la ligne c'est 2 , le triangle c'est $3 \ldots$ La vertu c'est la décade, le temps critique c'est 7 , le mariage c'est 5 , et la justice 4 ou 9 . On ne voit guère, à première vue, en quoi cette mystique des nombres peut concerner le mouvement et sa causalité. C'est le thème des accords musicaux qui a fait la transition, car les sons de la lyre sont dus aux vibrations de ses cordes, qui dépendent elles-mêmes de la longueur de telles cordes. Une corde deux fois moins longue vibre deux fois plus vite. Telle fut la découverte, ou la théorie afférente à un art déjà en possession de procédés tout à fait assurés, que nous devons aux Pythagoriciens. Les trois accords musicaux, octave, quinte, quarte, sont représentés respectivement par trois rapports numériques simples : $2 / 1,3 / 2,4 / 3$. Les nombres régissent donc les consonances obtenues avec la longueur relative des cordes. Les trajectoires des astres, distantes les unes des autres, ne sont-elles pas comme les cordes de la lyre ? Pythagore natif de Samos, à la fois savant et fondateur de communautés religieuses, est supposé avoir apporté à Crotone, où il fit sa résidence avant d'en être expulsé, la cosmologie milésienne. Cette cosmologie, telle qu'elle avait été constituée par Anaximène, fut modifiée par Pythagore. Ainsi l'air illimité est aspiré par le feu central, qui siège au centre de l'univers, et que les Pythagoriciens ont appelé symboliquement la mère des dieux. Les choses particulières se produisent, comme chez Anaximène, par condensation ou raréfaction. L'évolution de l'univers est cyclique et ce cycle est déterminé, du moins depuis Philolaos, l'astronome de l'Ecole, par la Grande Année, l'époque où toutes les étoiles et planètes ont repris leur position initiale. Comme chez Anaximandre, par ailleurs, il y a pluralité des mondes. Dans notre monde, c'est autour du feu central que tout s'ordonne et que se déroule la révolution circulaire des dix corps célestes. Le corps le plus éloigné (l'Olympe) est le ciel des fixes, dont le mouvement propre d'orient en occident aurait peut-être été reconnu pour la première fois par Pythagore lui-même. Puis se trouve le Cosmos, où se meuvent les cinq planètes, dont le mouvement est inverse de celui du ciel des fixes, le Soleil et la Lune. Enfin l'Ouranos est la région sublunaire où se trouve la Terre, qui tourne autour du feu central, ce qui produirait la succession des jours et des nuits. On voit que, pour les pythagoriciens, notamment chez Hicétas de Syracuse, la Terre n'est pas immobile ni le centre de l'univers. Entre le feu central et la Terre tourne également l'Anti-Terre, qui reste invisible pour nous; sa présence expliquerait les éclipses de la Lune, car c'est du feu central que la Lune et le Soleil reçoivent leur lumière, et ce dernier sa chaleur. La vitesse des astres varie avec leur distance, comme la vitesse des vibrations avec la longueur des cordes de la lyre. C'est pourquoi on doit retrouve $r$ dans le système céleste les consonances que nous connaissons. Le problème est que nous n'entendons pas cette "harmonie ». La raison de ce silence serait, pour les pythagoriciens, que nous y sommes habitués et qu'un son n'est perçu que par rapport à des silences.

b) L'éléatisme est assez différent du pythagorisme, bien qu'il soit né dans des lieux voisins. On considère comme son fondateur Xénophane de Colophon, venu, lui aussi, d'Asie Mineure au moment de l'invasion des Perses. Il aurait écrit un livre Sur la nature dont il nous reste 
quelques fragments. Citons les plus fameux : « Il n'y a qu'un seul Dieu, maître souverain des dieux et des hommes, qui ne ressemble aux mortels ni par le corps ni par la pensée »(frag.23), « Tout entier il voit, tout entier il pense, tout entier il entend. Mais, c'est sans aucun effort qu'il meut tout par la force de son esprit »(frag. 24 et 25). . Par ailleurs : " Il n'y eut dans le passé et il n'y aura jamais dans l'avenir personne qui ait une connaissance certaine des dieux et de tout ce dont je parle. Même, s'il se trouvait quelqu'un pour parler avec toute l'exactitude possible, il ne s'en rendrait pas compte par lui-même. Mais c'est l'opinion qui règne partout». Ces deux thèses font irrésistiblement penser aux deux «voies » de Parménide, c'est pourquoi on a fait de ce dernier le disciple de Xénophane. Dans les fragments du poème de Parménide qui nous sont parvenus, on trouve, en effet, mise dans la bouche de la déesse Dikè (Justice) qui s'adresse au jeune homme avide de savoir, une séparation très nette de ces deux voies: «Ecoute et retiens mes paroles qui t'apprendront quelles sont les deux seules voies d'investigation que l'on puisse concevoir. La première dit que l'Etre est et qu'il n'est pas possible qu'il ne soit pas. C'est le chemin de la Certitude, car elle accompagne la Vérité. L'autre, c'est :L'Etre n'est pas et nécessairement le Non-Etre est. Cette voie est un étroit sentier où l'on ne peut rien apprendre. Car on ne peut saisir par l'esprit le Non-Etre, puisqu'il est hors de notre portée ; on ne peut pas non plus l'exprimer par des paroles; en effet, c'est la même chose que penser et être » (frag.4-5). La première voie enseigne les attributs de l'Etre: il est inengendré, impérissable, indivisible, immobile et unique. On ne peut donc rien en apprendre pour une science du mouvement. Le problème est de savoir s'il faut condamner tout à fait la deuxième voie, que la déesse développe aussi dans la deuxième partie du poème. Il serait curieux que Parménide mette cette deuxième voie dans la bouche de la déesse, s'il n'y voyait que mensonge et illusion. Il serait peu vraisembable aussi que les anciens philosophes grecs aient attaché une certaine importance aux enseignements de cette deuxième voie, s'ils avaient pensé que Parménide n'y accordait aucune créance. Parmi toutes les opinions, certaines étaient probablement, pour Parménide, plus proches de la vérité que les autres, et ce sont celles-là qu'il a mises dans la bouche de la déesse. C'est pourquoi des interprètes récents choisissent de prendre au sérieux la deuxième partie du poème. Parmi eux, Jean Frère a montré qu'on a mal interprété les dires de la déesse. Le texte grec s'exprime ainsi :

(reproduire ici le texte en grec, tel qu'il est tiré de Die fragmente der Vorsakratiker, H.Diels, 1912,pp.158-159)

On traduit généralement de la façon suivante : «(Les hommes), par convention,, ont en effet assigné à deux formes des noms ; mais des deux une n'en est pas digne, et c'est en cela qu'ils se sont fourvoyés. Car ils ont estimé contraires leurs aspects, et leur ont assigné des signes qui fondaient leur distinction mutuelle. Des deux ; l'une est le feu étheré de la flamme, c'est le feu caressant et c'est le feu subtil, identique à lui-même en toutes directions, mais qui à l'autre forme identique n'est pas ; l'autre par son essence est l'exact opposé, c'est la nuit sans clarté, dense et lourde d'aspect.(frag. 8 ; trad. J.P.Dumont et alii, op.cit.p.263). En réalité, il faudrait traduire ce passage, selon J.Frère, de la façon suivante : "(Certains mortels) ont admis en leurs jugements, de nommer deux Formes; de ces deux Formes, il ne faut pas nommer seulement une unité - c'est de ce point de vue qu'on se trouve en errance. A l'opposé , (certains mortels) en séparèrent les structures, et leur attribuèrent des signes, en les mettant à part l'une de l'autre. D'un côté le feu éthéré de Flamme...et, à l'opposé, cette autre Chose prise aussi en elle-même, nuit sans clarté, structure épaisse et lourde $»^{3}$. Parménide veut dire dans ce passage, semble-t-il, que les deux contraires pythagoriciens Lumière/Obscurité sont à prendre en même temps, qu'ils se partagent l'empire du monde sensible sans s'exclure et que

\footnotetext{
${ }^{3}$ J.FRERE, Temps, désir et vouloir en Grèce ancienne, Editions Dioné, Paris-Athènes, 1995, p. 168
} 
l'erreur est d'en faire un couple d'opposés inconciliables. Il faut remarquer d'ailleurs que le fragment 12 fait référence à un certain mélange de la Lumière-Feu et de l'ObscuritéOpacité: «Les anneaux plus étroits sont remplis de feu sans mélange; viennent ensuite les anneaux de la nuit et parmi eux se répand une partie de la flamme. Au milieu de ces anneaux se trouve la Divinité qui gouverne tout. Partout elle préside au terrible enfantement, poussant la femelle à s'unir au mâle, le mâle à la femelle ${ }^{4}$. On pourrait sans doute en dire davantage si les fragments de la deuxième partie du poème étaient plus nombreux et si les extraits de la doxographie qui comparent Parménide aux auteurs de son temps, éclairaient ces fragments de façon indubitable, mais il faut se résoudre à laisser cette voie de l'opinion dans un certain clair/obscur, qui contraste avec la netteté de la première partie. C'est ce contraste qui, dès les temps contemporains de Parménide, semble-t-il, a fait accuser Parménide de contradiction. N'est-il pas contradictoire, en effet, d'affirmer l'unité absolue de l'Etre et de prétendre tenir un discours vraisemblable, et même révélé par une déesse, sur la multiplicité chatoyante des choses sensibles? Les naturalistes décidés qui multipliaient les principes contraires de la réalité sensible et les faisaient jouer dans un mouvement éternel n'étaient-ils pas plus cohérents ? C'est pour répondre à de telles objections, selon Platon, que Zénon d'Elée, disciple de Parménide, aurait inventé ces fameux arguments dirigés les uns contre l'hypothèse de la multiplicité, les autres contre l'hypothèse du mouvement. Zénon voulait montrer que les adversaires de son maître, qui l'accusaient de contradiction, tombaient dans des contradictions bien pires ${ }^{5}$. On ne peut s'étendre ici sur les premiers arguments qui concernent la multiplicité, mais la mention des seconds qui concernent le mouvement est indispensable.

Les arguments de Zénon contre l'existence du mouvement ont été rapportés par Aristote (Physique, VI, 239 b 5 - 240 a 18) et seulement par lui. Il est donc impossible de les envisager en dehors de l'ordre que leur a donné Aristote et que les commentateurs d'ordinaire considèrent comme authentique. Selon cet ordre, les deux premiers arguments se placent dans l'hypothèse de la divisibilité infinie du continu spatial et temporel, les deux derniers dans l'hypothèse contraire d'indivisibles d'espace comme de temps. Comme l'on sait, par un auteur ancien qui déclare tenir ce jugement d'Aristote, que Zénon « est l'inventeur de la dialectique », il est permis d'imaginer que Zénon fait face ici à un contradicteur qui , se trouvant vaincu sur la première hypothèse, maintenue dans le cadre un peu plus riche du second argument, se place dans la deuxième hypothèse, où Zénon parvient, là encore, en deux étapes, à le réduire à la contradiction. Le premier argument a reçu le nom de la Dichotomie. Il s'énonce comme suit : "Avant qu'un corps en mouvement puisse atteindre un point donné, il doit d'abord traverser la moitié de cette distance ; avant qu'il puisse atteindre cette moitié, il doit traverser le quart, et ainsi indéfiniment ». A une infinité d'espaces à parcourir, correspond une infinité de temps, si bien que la traversée de la distance entière est impossible. On imagine alors l'adversaire voulant tourner la difficulté en invoquant la situation banale où un mobile, qui va plus vite qu'un autre, dépasse ce dernier. Dans ce cas une distance est bien parcourue, et même dépassée. Zénon répond à l'adversaire par l'argument, dit l'Achille : « Si la tortue a de l'avance sur Achille, celui-ci ne pourra jamais la rattraper, quelle que soit sa vitesse ; car pendant qu'Achille court pour atteindre le point d'où est partie la tortue, celle-ci avance de telle sorte qu'Achille ne pourra jamais annuler cette avance, si minime soit-elle ». On voit que c'est encore la divisibilité infinie de l'espace et du temps qui permet à Zénon de l'emporter sur son adversaire. On peut supposer alors que ce dernier ait eu l'esprit de présenter une objection qui se place dans la seconde hypothèse, celle où on ne peut diviser à l'infini l'espace et le temps, mais où on se heurte à des indivisibles, en particulier l'instant s'il s'agit du temps. Zénon aurait alors avancé son troisième argument, la Flèche: «La flèche

\footnotetext{
${ }^{4}$ cf.J.P.Dumont et alii, p. 263

${ }^{5}$ PLATON, Parménide, $127 \mathrm{a}-128$ e
} 
lancée est toujours immobile; en effet, tout corps est soit en mouvement, soit en repos ; et il est en repos quand il se trouve dans un espace égal à son volume; or la flèche, se trouve, à chaque instant, dans un espace égal à son volume "). Vaincu sur le terrain d'un zéro de durée, l'adversaire se serait rabattu sur l'hypothèse d'un instant étendu en durée, mais de durée minimale, pendant laquelle la flèche pourrait parcourir un minimum d'espace. Zénon aurait alors répliqué à son adversaire par l'argument du Stade: »Quand des masses égales se déplacent, à même vitesse, les unes dans un sens, les autres dans le sens contraire, le long de masses égales et qui sont immobiles, le temps que mettent les premières à traverser les masses immobiles est égal au double du même temps ». Il faut supposer ici que les masses, toutes de même longueur, sont indivisibles, et que les masses mobiles sont disposées de part et d'autre des masses immobiles, de telle sorte qu'elles partent toutes de la même ligne qui coupe les masses immobiles en deux parties égales, et que pendant qu'elles traversent une certaine quantité (soit 2) de masses immobiles, elles croisent la quantité double (soit 4) de masses mobiles, si bien qu'une même quantité prétendument indivisible de temps (soit $2 / 2=1$ indivisible) est égale à son double (soit $4 / 2=2$ indivisibles). On verra bientôt qu'Aristote s'est efforcé de réfuter, et avec un certain succès, ces quatre arguments. Il est certain que ces arguments visaient à nier l'existence même du mouvement en tant que déplacement dans l'espace et le temps, et que les commentateurs qui prétendent que Zénon ne visait que les pythagoriciens attachés (à cause de leur théorie des nombres entiers) à la discontinuité de l'espace et du temps, ne tiennent pas compte de la disposition des quatre arguments. Ce qui est vrai, c'est qu'on ne peut analyser le mouvement sans se donner une représentation adéquate de l'espace et du temps, ce qu'Aristote, on le verra, saura retenir.

\section{3. les physiologues au temps de Socrate}

On sait que Socrate, qui imprima un nouveau tour à la philosophie grecque, ce dont s'indigna Nietzsche à la fin de notre $\mathrm{XIX}^{\mathrm{e}}$ siècle, a pu s'informer directement de toute la littérature physiologique (au sujet de La Nature) du $\mathrm{V}^{\mathrm{e}}$ siècle, puisqu'il mourut en 399 (av.J.-C.). Ces physiologues du $\mathrm{V}^{\mathrm{e}}$ siècle, comme on les appelle, s'efforcèrent sans doute d'éviter les difficultés de l'éléatisme, tout en profitant des progrès que le pythagorisme avait fait faire à l'astronomie. Ce faisant, ils se distinguèrent par l'originalité, comme c'est le cas d'Héraclite d'Ephèse, d'Anaxagore de Clazomène et d'Empédocle d'Agrigente, ou même fondèrent une nouvelle Ecole, avec Leucippe et Démocrite.

a) Héraclite d'Ephèse est traditionnellement opposé à Parménide, comme le philosophe du Devenir au philosophe de l'Etre. Cette tradition ne se trompe pas, en tant que le premier porta l'accent sur l'impermanence des choses, tandis que le second, avant lui, avait mis l'accent sur la permanence de l'Etre. Mais leurs figures, autant qu'elles peuvent être restituées à partir des témoignages qu'on a d'eux, ne présentent pas un contraste aussi accusé. D'un côté nous avons vu que Parménide avait su faire son profit des spéculations pythagoriciennes sur la Lumière et la Nuit, d'un autre côté Héraclite, qui voyait tout sous l'empire du Logos, qui semble être pour lui la raison universelle, reconnaissait le primat de l'unité sur la diversité. Qu'on en juge par ces fragments: "L'Un, le seul sage, ne veut être appelé et veut le nom de Zeus » (frag. 32 ); «Un est le savoir ; il connaît la pensée, par qui sont gouvernées toutes choses au moyen de toutes choses» (frag. 41) : «La loi aussi, obéir à la volonté de l'Un » (frag.33); « Pour Dieu, toutes choses sont belles, bonnes ett justes; mais les hommes ont forgé l'idée que certaines sont injustes et d'autres justes » (frag. 102) ${ }^{6}$. On n'est pas loin, à en juger par

${ }^{6}$ J.P.Dumont et alii, op.cit.,pp. 154,155, 169 
ces fragments, de la dualité entre la voie de la vérité et la voie de l'opinion. Il est possible qu'Héraclite ait voulu les unir, mieux que Parménide y était parvenu. Il reprend la doctrine pythagoricienne des contraires, tout en désapprouvant la «polymathie » de Pythagore. Mais ces contraires sont inclus dans l'unicité du Logos : «La route, montante et descendante, une et même » (frag.60). Quels sont ces chemins qui décrivent les mêmes processus en sens inverse ? Diogène Laërce en livre une description : «Parmi les contraires, celui qui conduit à la génération est appelé guerre et discorde ; celui qui conduit à l'embrasement concorde et paix. Le cangement est une route montante-descendante et l'ordonnance du monde se poursuit selon cette route. En se condensant, le feu s'humidifie; et, en se resserrant plus encore il engendre l'eau; et quand l'eau cristallise, elle se change en terre. Telle est la route descendante. A rebours,la terre se liquéfiue, d'elle naît l'eau et de celle-ci les autres éléments. Il ramène presque toutes choses à l'évaporation à partir de la mer. C'est la route montante. Des exhalaisons naissent de la terre et de la mer, les unes claires et pures, les autres obscures. Le feu se trouve alimenté par les exhalaisons claires, l'humide par les autres. Il n'indique pas clairement la nature de l'enveloppe terrestre... (IX) $\gg^{7}$ Immédiatement avant ce passage, Diogène Laërce a fait allusion à l'embrasement, par lequel le feu renouvellerait, après chaque période de conflit des contraires, l'univers dans sa totalité : «L'univers, selon lui, est limité et il n'y a qu'un monde, qui a été créé par le feu et qui retournera au feu après certaines périodes, éternellement. C'est le destin qui le veut ainsi ». Cette idée de l'éternel retour (qui diffère de la Grande Année pythagoricienne) sera reprise par les stoïciens et certains commentateurs récents ${ }^{8}$ pensent que Diogène Laërce a ajouté, de lui-même, cette idée à l'héritage authentique d'Héraclite. Mais tel n'était pas l'avis de Léon Robin, qui s'appuyait sur le témoignage d'Aristote (Physique, 205 a 3). Dans ce dernier passage, Aristote ne dit rien de plus qu'Héraclite dans le fragment 66 : « Sur toutes choses le feu ayant fondu, il les jugera et $s^{\prime}$ en saisira ${ }^{9}{ }^{9}$. Mais le fragment 30, semble bien indiquer que les embrasements sont périodiques, puisque l'univers est éternel, et que le feu est son élément principal qui alternativement s'allume et s'éteint : "Ce monde-ci, le même pour tous, nul des dieux ni des hommes ne l'a fait. Mais il était toujours, est et sera feu éternel, s'allumant sans cesse et s'éteignant en mesure " ${ }^{10}$. On a déjà vu, selon le témoignage de Diogène Laërce, que la création s'opérait grâce au conflit des contraires. Les fragments connus d'Héraclite confirment bien ce témoignage : "L'opposé est utile et des choses différentes naît la plus belle harmonie; et toutes choses sont engendrées par la discorde »(frag. 8$)^{11}$; et aussi : "Conflit est le père de tous les êtres, le roi de tous les êtres; aux uns il a donné forme de dieux, aux autres d'hommes. Il a fait les uns esclaves, les autres libres » (frag.53) ${ }^{12}$. Tout est donc réglé par le jeu des contraires, mais cela n'enlève rien au jeu du hasard, au caractère imprévisible des événements, qui maintient tout sous la quasi-loi au moins aussi puissante du devenir : «Le temps est un enfant qui s'amuse, il joue au trictrac. A l'enfant, la royauté » (frag. 52); «Dans les mêmes fleuves, nous entrons et nous n'entrons pas; nous sommes et nous ne sommes pas » (frag.49 a); "On ne peut entrer deux fois dans le même fleuve » (frag.91). De ces deux derniers fragments, il semble que le premier vise l'impermanence du moi et le second l'impermanence du fleuve, si bien que le devenir est universel. Sur ce point au moins, la tradition qui a prédominé dans la mémoire qu'elle a gardée d'Héraclite, semble ne s'être pas trompée.

\footnotetext{
${ }^{7}$ ibid.,p. 131

${ }^{8}$ c'est le cas de Barbarin Cassin dans une conférence donnée à Strasbourg dans les années 80

9 ibid.,p. 161

${ }^{10}$ ibid.p. 153

${ }^{11}$ ibid.p. 147

${ }^{12}$ ibid. p. 158
} 
b) Anaxagore de Clazomène naquit vers l'an 500 (av.J.-C.) en Asie Mineure. Il dut sans doute s'exiler à Athènes, où il devint l'ami de Périclès, mais ne jouit guère de la gloire de ce dernier, puisqu'il fut condamné, après la disgrâce ou la mort de son protecteur, pour impiété (il avait considéré les astres comme des pierres (incandescentes), ce qui était conforme à l'esprit des philosophes ioniens) et mourut en exil. Son originalité consiste à poser une infinité d'éléments, par quoi il est fort différent des Ioniens, même d'Anaximandre. Ces éléments sont appelés « semences » et ils sont si universellement distribués qu' on doit dire que « toute chose possède une portion de toute chose " (frag. 11 et 12), formule qui a connu une grande fortune sous la forme populaire : " tout est contenu dans tout». La formule serait paradoxale si Anaxagore n'avait ajouté que chaque chose est désignée par les éléments qui sont en elle les plus nombreux (frag. 12, ad finem). A l'origine donc, les semences de toutes choses existaient en désordre. Qu'est-ce qui les a séparées et rassemblées dans des agrégats plus ou moins homogènes ? C'est l'Intellect, qu'Anaxagore appelle le nouset qui est distinct de toutes les semences. L'action de l'Intellect est décrite dans la majeure partie du fragment 12 : «Toutes les choses qui ont une âme, qu'elles soient grandes ou petites, sont toutes sous le pouvoir de l'Intelleç̃tC'est l'Intellect qui a exercé son pouvoir sur la révolution universelle, de telle sorte que c'est lui qui a donné le branle à cette révolution. Le point de départ de la révolution fut petit; ensuite celle-ci s'accroît et elle s'accroîtra toujours davantage. Et l'Intellect a connu toutes les choses, aussi bien celles qui sont mélangées que celles qui sont discriminées et séparées ; et ce qui devait exister aussi bien que ce qui existait, et tout ce qui n'existe pas maintenant aussi bien que tout ce qui existe maintenant et ce qui existera, tout cela fut ordonné par l'Intellectet aussi cette révolution, que suivent à présent les astres, le soleil et la lune, et l'air et l'éther qui résultent de la discrimination. C'est cette même révolution qui a produit la discrimination. Le dense se constitue par discrimination à partir du rare, le chaud à partir du froid, le brillant à partir du lumineux, le sec à partir de l'humide. Les multiples choses ont des parties multiples; et nulle chose n'existe d'une manière discriminée ou totalement séparée d'une autre chose, excepté l'Intellecț'Intellect est tout entier semblable à lui-même, à la fois grand et petit $»^{13}$. Ainsi l'Intellect est la cause motrice de tout ce qui se trouve organisé dans le monde, il connaît ce qu'il fait, mais il n'a pas de plan : ce qui devait décevoir Socrate et scandaliser Platon. L'Intellect, bien que distinct des semences, est, lui aussi, matériel : " C'est de toutes les choses la plus légère et la plus pure. Il ( le nous) possède toute espèce de connaissance de tout et la force la plus grande » (in frag. 12). La philosophie d'Anaxagore semble être une forme de vitalisme, où la force du nousest incomparable, mais dont l'action est purement mécanique. En cela il est bien l'héritier du mécanisme et de l'animisme ioniens.

c) Empédocle d'Agrigente est l'exact contemporain d'Anaxagore et les historiens de la philosophie placent indifféremment l'un avant l'autre. Ce fut un personnage célèbre de la Sicile ; c'est pourquoi on le compare souvent au pythagorisme et à l'éléatisme dont il connaissait bien les principales thèses. Du pythagorisme il garde la croyance à la métempsychose, cette destinée des âmes qui, selon Empédocle, ont donné leur foi à la «Discorde furieuse », dont on verra le rôle cosmologique. De l'éléatisme il partage la conviction de l'unité de l'Etre étendu et fini, ainsi que le caractère relatif de ce que nous appelons « génération » et « corruption », mais il introduit dans cette étendue finie la pluralité et le mouvement d'une façon explicite (sans aller jusqu'à la pluralité des mondes comme le faisaient Anaximandre et Anaxagore). Pour lui, les « racines » des choses sont au nombre de quatre : le feu, l'eau ; l'éther (plus rarement désigné par le nom d'air), la terre. Il n'entreprend

13 ibid. p. 135 
pas de dériver ces éléments les uns des autres, comme le faisaient les Ioniens. Il est donc l'ancêtre de la doctrine des quatre éléments, qui aura une longue postérité, puisqu'elle ne fut ébranlée que par les recherches d'alchimie au Moyen-Age et ensuite par la physico-chimie moderne. Cette doctrine apparaît plus vraisemblable que celle d'Anaxagore, en ce qu'elle refuse que les tissus organiques, comme l'os et la chair (ce qu'Aristote appellera les « homéomères »), soient déjà contenus, sous forme infinitésimale, dans les « semences", mais les conçoit comme formés des quatre éléments (l'os contenant, par exemple, quatre parties de feu, deux parties de terre et deux parties d'eau). Récemment Denis O'Brien a présenté une interprétation d'ensemble de la cosmologie d'Empédocle, qui restaure la vision cyclique de l'auteur, mise à mal par le néo-platonisme et les commentateurs plus récents qui suivent cette tradition. Il montre que la cosmologie d'Empédocle est très différente de celle d'Anaxagore qui, probablement, l'a précédée de peu : «Les quatre « racines » sont soumises à l'influence, non pas d'un seul « moteur » (l'Intellect), mais à deux pouvoirs opposés : la Discorde, principe de mouvement et de pluralité, s'oppose à l'Amour, cause de l'unité et du repos. Ces deux pouvoirs, l'Amour et la Discorde, conformément aux termes qui leur sont imposés par un puissant serment, se partagent, à titre égal, la vie de l'Univers. Les quatre « racines » sont en effet tantôt rassemblées par l'Amour en une unité homogène et immobile, tantôt soumises à la séparation et au mouvement sous l'influence de la Discorde, la période d'unité et de repos étant égale en durée à la période de séparation et de mobilité » ${ }^{14}$. Tel est le schéma général, mais il n'implique pas que, en chaque demi- cycle, l'Amour travaille sans la présence de la Discorde, ni vice versa. C'est progressivement que l'un gagne sur l'autre, comme l'explique le fragment 35: « Quand au plus profond du tourbillon la Haine fut tiombée, que l'Amour atteignit le centre du remous, les éléments alors de concourir en lui, touys pour faire un seul Un, non pas sur le champ, mais avec réflexion, , venant qui d'un côté et qui venant de l'autre. Des éléments ainsi mélangés s'écoulèrent des milliers et milliers de races de mortels. Mais de nombreux objets restèrent non mêlés, séparés à côté des choses mélangées, tous ceux que Haine encore en suspens retenait . car elle ne s'était pas encore, sans reproche, retirée jusqu' aux bords extrêmes du cercle ; ici elle restait dans les membres, et là elle en était sortie. Mais plus elle fuyait de façon continue, toujours plus s'avançait le bénéfique élan immortel de l'Amour exempt de tout reproche. Aussitôt devenaient mortelles désormais les choses qui naguère avaient connu les joies de l'immortalité ; et les choses jadis non mêlées se brassaient, changeant de direction. Des éléments ainsi mélangés s'écoula une pluralité proprement infinie de races de mortels, étonnantes à voir $\rangle^{15}$. Chaque demi-cycle est donc différent, selon qu'il commence à partir du règne prolongé de l'Amour, ou à partir du règne dévastateur de la Discorde, D.O'Brien distingue, en conséquence, deux zoogonies qu'à la suite des indications d'Empédocle, il décrit en ces termes : " A l'aube du monde issu d'une période d'unité et de repos, surgissent des êtres possédant un lot égal de feu et d'eau, les deux éléments dont la prédominance est constitutive, respectivement, de 1 'animal mâle et de l'animal femelle. Ce sont donc des êtres bisexués (ou asexués), lesquels soumis à l'influence grandissante de la Discorde, ne peuvent plus maintenir leur unité primitive et se voient contraints de se scinder en hommes et femmes. Dans le monde qui se forme à la suite de la séparation totale des "racines », le procédé est plutôt l'inverse. Dans un premier temps sont issus de la terre des membres isolés (des têtes sans cou, des bras privés d'épaules, des yeux privés de tête...),lesquels, sous l'influence grandissante de l'Amour, se réunissent, d'abord pour former des monstres (des êtres «à double visage et à double poitrine », des corps humains à tête de bœufs, des créatures douées de "mains innombrables »...) pour devenir

${ }^{14}$ D.O’Brien, « Empédocle » dans Le Savoir Grec, dictionnaire critique s .1 dir. de J.Brunschwig, G. Lloyd et P.Pellegrin, Flammarion, 1996 , p. 635

${ }^{15}$ J.P.Dumont et alii, pp.388-389 
ensuite des hommes et des femmes » ${ }^{16}$. Ici ont été décrites les premières étapes de l'une et l'autre zoogonie, on imagine facilement que les deuxièmes étapes s'avancent vers les résultats d'où sont partis les premières. Il fallait une grande imagination à Empédocle pour unir à la rigueur qu'il tenait de Parménide, à qui il emprunte la forme sphérique de l'Etre total, un dynamisme vital, dont ses prédécesseurs avaient eu quelque idée, mais qu'ils n'avaient jamais pu décrire avec quelque vraisemblance, prisonniers qu'ils étaient de procédés mécaniques, auxquels Empédocle substitua les causes motrices de l'Amour et de la Discorde.

d) Leucippe et Démocrite rédigèrent leurs traités dans le dernier tiers du $V^{e}$ siècle. Le premier aurait été un disciple de l'Ecole d'Elée, qui aurait ouvert une école à Abdère, où Démocrite devint son disciple. Il est malaisé de distinguer leurs travaux qui, pour Aristote, expriment une même doctrine. Comme celle d'Empédocle, leur doctrine évite les difficultés de l'éléatisme en combinant, bien que différemment, l'un et le multiple. L'un n'est plus la sphère de Parménide ou d'Empédocle, mais l'être qui est divisé d'emblée en une infinité d'atomes (souvenir de l'infinité d'Anaxagore ?), tous indivisibles. Le multiple, qui était le non-être pour Parménide, est ici le vide infini, grâce auquel les atomes peuvent se mouvoir, s'entrechoquer, s'assembler et se séparer, bref constituer des mondes, où ces phénomènes se reproduisent, et font naître, par le hasard qui ne se distingue pas ici de la nécessité, des êtres vivants. Il y a même des dieux, composés d'atomes très subtils, qui se meuvent entre les mondes. On ne peut qu'être frappé par l'audace de cette philosophie de la nature, qui supprime toute trace d'animisme, bien qu'elle reconnaisse l'existence d'âmes composées, comme les dieux, d'atomes subtils, et présente un mécanisme strict. Ce mécanisme sera repris par le mécanisme européen du $\mathrm{XVII}$ siècle et inspira ensuite la théorie atomique, évidemment différente, des chimistes et cristallographes du $\mathrm{XIX}^{\mathrm{e}}$ siècle. De nos jours, les constituants qu'on appelle les atomes ne sont plus insécables, mais composés d'un noyau (qui leur procure leur masse) et d'électrons qui leur permettent de s'unir entre eux. Les atomes de Démocrite, de leur côté, ne diffèrent que par leur figure, leur ordre, leur position et leur grandeur (qui peut varier); il n'était pas question, semble-t-il de leur conférer une masse qu'ils ne pouvaient acquérir que par leur agrégation. S'ils se rencontrent, c'est parce que leurs trajectoires se coupent et créent des entrelacements et des agrégats d'atomes. Grâce à des tourbillons, qu'utilisaient déjà les Ioniens et Anaxagore pour séparer les assemblages hétérogènes et rassembler les éléments semblables, les atomes de même forme et de même grandeur se rapprochent et la vision ionienne ou pythagoricienne des étagements d'éléments se trouve expliquée. Ce type d'explication demeure cependant purement intellectuel, car les atomes sont invisibles. C'est probablement ce caractère purement idéal du mouvement des atomes qui fera, au long des siècles, le prestige de la doctrine. Ce qui est intelligible s'oppose ici au sensible. Mais l'intelligible est réel, tandis que les qualités comme le doux, l'amer, les couleurs etc... ne sont que des " conventions » dépendantes de nos appareils sensoriels. Il y ici une réminiscence de l'opposition éléate entre la vérité et l'opinion, mais aussi la préfiguration de la distinction, apparue chez les mécanistes du XVII ${ }^{\mathrm{e}}$ de notre ère, entre les " qualités premières » (étendue, forme, masse, trajectoire) et les "qualités secondes» (couleurs, odeurs, saveurs). C'est pourquoi l'atomisme démocritéen a joué le rôle d'un modèle de connaissance physique, purement mécanique et déterministe. L'ajout qu'y fera Epicure, au $\mathrm{III}^{\mathrm{e}}$ siècle avant notre ère du clinamen ou déviation imprévisible des atomes durant leur chute dans le vide, apportera à ce modèle déterministe une part de contingence, mais il n'entamera pas le mécanisme foncier, bien que caché aux regards, des phénomènes visibles. L'atomisme ancien et l'épicurisme sont, par là, bien éloignés de la science moderne, bien qu'ils l'aient inspirée, et leur fortune actuelle réside davantage dans l'attitude morale qu'ils préconisaient : une grande modération

${ }^{16}$ D.O’Brien, dans Le Savoir Grec, op.cit.p. 637 
qui vise à ne pas détruire les équilibres fragiles et à ne pas compromettre les réussites de la vie, là où elles ont eu la chance d'apparaître. Que les disciples d'Epicure se soient réunis dans un Jardin est certainement conforme à la leçon d'humaine prudence qu'ils tiraient d'un naturalisme dépouillé de toute croyance magique ou religieuse.

\section{Platon et Aristote}

Avec Platon et Aristote, l'enquête atteint les deux philosophes les plus célèbres de l'Antiquité gréco-romaine, ceux qui n'ont jamais souffert d'un oubli prolongé, bien que leur renommée, comme il est inéluctable, ait été sujette à des fluctuations. D'une certaine façon, c'est le $\mathrm{XIX}^{\mathrm{e}}$ siècle qui a remis les Présocratiques à l'honneur, malgré l'état déplorable où se trouve la connaissance que nous en avons. Dès le début du siècle, Hegel avait réhabilité Héraclite et Nietzsche lui a plus tard emboîté le pas, en n'omettant pas d'y ajouter Empédocle, le noble sicilien qui croyait à l'éternel retour. En comparaison de ces auteurs exhumés d'une tradition classique peu généreuse à leur égard, Platon et Aristote ne pouvaient apparaître que plus banals, moins poétiques et moins romantiques, trop marqués par la figure de Socrate, dont ils dépendaient, et qu'ils étaient accusés d'avoir canonisée comme l'exemple à suivre. En fait ni Platon ni Aristote ne sont morts martyrs de la philosophie, bien que le premier ait parfois payé fort cher d'avoir tenté d'appliquer sa pensée à la politique de Syracuse, et que le second ait été accusé faussement d'impiété, ce qui le contraignit à l'exil où il ne survécut pas longtemps. L'important c'est qu'avec Platon et Aristote la philosophie s'est implantée pour des siècles à Athènes, où l'Académie et le Lycée ont transmis, serait-ce au prix de déformations, la doctrine de leurs fondateurs. Ces institutions veillèrent en tout cas sur la conservation des œuvres de ces fondateurs disparus. Nous possédons presque tous les Dialogues de Platon et presque tous les cours écrits d'Aristote. Ces œuvres sont ainsi prêtes pour de continuelles renaissances, et l'érudition contemporaine a apporté une contribution notable à la mise en valeur de ces vénérables textes, parmi lesquels ceux qui concernent le mouvement font apparaître des nouveautés très remarquables par rapport aux doctrines antérieures.

a) Platon partageait certainement la position qu'il prête à Socrate dans le Phédon, à savoir que la conception de l'Intelligence comme cause motrice chez Anaxagore est fort décevante (98 a$100 \mathrm{~d}$ ), car elle est mécanique, et non, comme on aurait pu s'y attendre, téléologique, c'est-àdire empreinte de finalité. La cause pour laquelle Socrate est sur son lit dans sa prison, c'est que les Athéniens ont jugé bon de le condamner, et lui de subir cette condamnation, plutôt que de quitter Athènes, où il a toutes ses raisons de vivre. On ne peut s'écarter de cette cause principale sans tomber dans des explications savantes, qui diffèrent d'un auteur à l'autre, sans qu'elles atteignent le motif essentiel. On peut croire que Platon lui-même a réfléchi sur les explications des «physiologues» et les a trouvées arbitraires, dépendantes d'un modèle mécanique incessamment retouché, selon les éléments matériels que ces auteurs retenaient comme primordiaux. Or, pour Platon, l'explication par le meilleur conduit, par l'intermédiaire d'une réflexion sur l'art de l'artisan, à l'explication par la forme, c'est-à-dire par le modèle que l'artisan a dans sa tête, comme il est dit dans le Cratyle (389 ab). Si l'on rapproche ce dernier dialogue du Phédon et du Ménon, il apparaît que toute production et tout mouvement dépendent de formes intelligibles qui en fournissent le modèle, c'est-à-dire d'Idées qui sont statiques, à la différence des choses sensibles prises, comme le disait Héraclite, dans un perpétuel écoulement. Ce perpétuel écoulement ne peut pas être objet de science, seules les formes intelligibles le sont. Telle est la première formulation de la théorie des Idées. Cependant Platon s'aperçut que cette métaphysique du suprasensible était pleine de périls et 
qu'il ne fallait pas considérer les Idées comme des blocs séparés. Il y a une vie, une connaissance, qui va de l'une à l'autre et qui les fait apparaître comme des relations. Cette deuxième étape de la théorie des Idées est exposée dans le Sophiste, où le royaume des Idées est dominé par cinq grands genres : l'être, le même, l'autre, le repos et le mouvement ( $254 \mathrm{~d}-$ 255 e). Le mouvement est ici introduit au sommet de l'être par la vie et la connaissance qui doivent exister, de façon éminente, dans le suprasensible. Nous avons affaire ici à une " métaphysique » originale, mais qui n'éclaire pas en elles-mêmes les choses de la nature, dites seulement « participer » au monde intelligible. Or il fallait savoir quelque chose de cette "participation", découverte mais non expliquée par cette ressemblance assez lointaine que les choses sensibles entretiennent avec les Idées intelligibles. Cette ressemblance, on l'a vu, avait déjà frappé les pythagoriciens, qui voyaient des nombres partout, en particulier dans les mouvements célestes. Il n'est donc pas étonnant que Platon, vers la fin de sa vie, ait emprunté à l'un d'entre eux, qui y joue le rôle principal, le titre de son ouvrage cosmologique, le Timée ou De la Nature. Dans ce dialogue, en effet, l'astronome Timée raconte la genèse de l'Univers. Il est frappant qu'en la matière le narrateur ne prétend pas offrir un discours d'une exactitude absolue. L'exactitude, en effet, est la propriété du plan intelligible de l'être, sur le modèle duquel a été fabriqué l'Univers. Mais «ce qu'est au devenir l'être, au croyable l'est la vérité » $(29$ c). Seul le vraisemblable donc peut être atteint en cosmologie. Il y a ici comme une réminiscence de Parménide, sur lequel Platon n'ose porter une main "parricide » que dans le dialogue du même nom. L'être de Platon est beaucoup plus riche, on l'a vu, que "l'un » de Parménide, et de la même façon le devenir de Platon sera beaucoup plus compliqué et assuré que la fresque parménidienne de la " deuxième voie »; cependant, Platon s'accorde avec Parménide sur le caractère incertain de la cosmologie, qui est comme la " deuxième voie » de Platon. Cependant, de même que l'être, chez Platon, peut se multiplier grâce à la définition du non-être comme autre ou différent, de même le mouvement peut être sauvé, non seulement parce que l'idée en réside, on l'a vu, dans le monde intelligible, mais parce que la raison même qui avait conduit, on l'a vu, à l'introduire dans le monde intelligible se retrouve, à moindre échelle, mise en œuvre, grâce au Démiurge, dans le monde sensible, à savoir la création de 1 'Ame du Monde. Ce point est tout à fait propre à Platon, et c'est le mérite, il y a un quart de siècle, de Luc Brisson d'avoir montré que ce n'était pas, chez Platon, une invention tardive : "Que l'âme, en général, soit principe de mouvement, ce n'est pas, malgré l'avis de plusieurs interprètes, une découverte tardive de Platon. En effet, on retrouve cette doctrine dans le Charmide (156 e), dans le Gorgias (465 c-d), dans le Phédon (80 a ; 94 b-e), dans le Phèdre (246 b). Les Lois (896 e - 897a) n'innovent pas, elles récapitulent $"{ }^{17}$. Ce qui est remarquable, dans le Timée, c'est que la doctrine de l'âme automotrice, depuis longtemps exprimée, y prend, pour le monde sensible, sa forme définitive. Le même auteur apporte, en effet, les précisions suivantes: "Etant source du mouvement, l'âme doit absolument être automotrice (Phèdre, 245 c - 246 a). En effet, on ne peut remonter indéfiniment dans la chaîne du mouvement ni en ce qui concerne l'ordre causal ni en ce qui concerne la temporalité. Si on cherchait un principe pour le mouvement de l'âme, on devrait le trouver dans un mouvement d'un ordre plus élevé ou dans une réalité échappant au mouvement. L'être du mouvement de l'âme dépend, bien sûr, de la forme intelligible du mouvement qui est éternelle, mais le mouvement propre de l'âme ne dépend de rien qui n'est pas mouvement. Par ailleurs, on ne distingue nulle part dans le Timée un mouvement supérieur à celui de l'âme, et qui en serait la source. L'âme apparaît donc comme automotrice : omettre ou contester cette définition conduirait à mutiler une bonne partie de la doctrine platonicienne. Alors, en effet, il faudrait affirmer que l'immortalité de l'âme, qui

${ }^{17}$ L.Brissson, Le même et l'autre dans la structure ontologique du Timée de Platon, Paris, Editions Klinksieck, 1974, p.406 
repose sur son automotricité (Phèdre, $245 c-d$ et plus spécialement 245 c 5), n'a jamais été soutenue par Platon ou a été abandonnée à un moment ou à un autre ${ }^{18}$.

Peut-on réduire à cette âme automotrice, d'où provient toute âme, chez l'animal comme chez l'homme, la conception platonicienne du mouvement ? Ce serait oublier que le Démiurge, tout en fixant son regard sur le Modèle intelligible, travaille sur un matériau préexistant à sa fabrication. Or ce matériau, ou milieu spatial, est-il mobile ou immobile ? Il n'est pas mobile d'un mouvement régulier, comme le sont les mouvements de l'Ame du monde sur les cercles du même et de l'autre qui guident les astres et les planètes, mais il est perpétuellement agité et cette agitation permet même aux quatre éléments (terre, eau, air, feu) de se former, à partir de surfaces triangulaires qui forment quatre sortes de polyèdres réguliers correspondant aux quatre éléments (respectivement le cube, l'icosaèdre, l'octaèdre et le tetraède). Le cinquième polyèdre régulier, qu'avaient découvert les mathématiciens du temps de Platon, est le dodécaèdre, dont Platon a fait le modèle qu'a pris le Démiurge pour réaliser « le tableau de l'Univers » (le dodécaèdre est voisin de la sphère et choisi aujourd'hui par certains cosmologues qui y voient une structure convenable pour la topologie de l'Univers). Il est intéressant de remarquer ici que cette composition des quatre éléments permet à Platon d'expliquer pourquoi la terre, qui est formé de triangles rectangles isocèles capables de constituer des cubes, se mélange aux autres éléments, sans se transformer en eux, à la différence des trois autres éléments qui, formés de triangles rectangles scalènes, se prêtent à toutes sortes de combinaisons, dont les transmutations. Platon est donc, sur ce terrain, héritier des Ioniens, d'Empédocle aussi d'une certaine façon, mais également de Démocrite, car ces triangles, dont les formes et les assemblages varient, font penser aux « atomes » de Démocrite et obéissent, comme ces derniers, à la nécessité, c'est-à-dire au jeu de causes mécaniques qui agissent au hasard. Il y a donc du mouvement dans le milieu spatial et Platon ne dit pas d'où il vient. On sait seulement qu'il ne vient pas de l'Ame du Monde. Cependant, de même que les figures géométriques qu'il agite, il n'échappe pas au contrôle du monde intelligible d'un côté, du Démiurge et de l'Ame du Monde qui en prennent possession de l'autre. Platon en parle comme d'une " cause errante ». Par rapport à l'action du Démiurge, cette cause errante est dite « adjuvante », et par rapport à l'action de l'Ame du Monde, elle est dite " seconde ». Platon explique par là que l'ordre qui règne dans l'Univers n'est pas sans faille, puisque le Démiurge a fait ce qu'il a pu, et que l'Ame du monde, constitué par lui pour continuer son rôle directeur, doit composer, elle aussi, avec les contraintes du « réceptacle » qui est la " nourrice » des choses sensibles. Là se trouve l'origine du dualisme platonicien, qui sera accentué par la suite. Chez Platon, le dualisme de la causalité donne lieu à d'innombrables compromis, dont L.Brisson montre qu'ils fournissent la raison des phénomènes que les physiologues antérieurs s'acharnaient à expliquer : la division et la condensation. Platon, lui aussi, a donné son explication: "Nous avons vu que la sphère du monde enveloppe tout ce qui est corporel. Or les quatre éléments se répandent, à l'intérieur de cette sphère, en quatre couches concentriques. Ces quatre couches concentriques suivent donc la révolution circulaire impartie au tout. Mais, comme il n'y a pas de vide, d'une part, les particules ne peuvent s'épandre à l'infini vers l'extérieur; et, d'autre part, à l'intérieur elles ne peuvent circuler que dans les interstices toujours remplis tirant leur origine de l'absence d'homogénéité entre les divers éléments. Ce qui donne lieu à une réaction en chaîne dont Platon comprend le mécanisme par l'intermédiaire de la «compression impliquée par le processus du refoulement " (58 b 4-5). Et cette réaction en chaîne produit le processus décrit plus haut : les petites particules qui se glissent dans les interstices formés par les grosses particules, ou bien divisent ces grosses particules ou bien sont condensées par elles. C'est là l'origine du double

${ }^{18}$ ibid. pp.406-407 
mouvement de toute transformation corporelle : la division et la condensation ${ }^{19}$. En définitive, il y a toujours, dans le monde sensible, la double action des « causes nécessaires » et des «causes divines » : le physiologue doit tenir compte des deux, et l'homme sage se rappeler qu' « il faut rechercher en toutes choses les (causes) divines en vue d'acquérir une vie bienheureuse, autant que notre nature le comporte ; les causes nécessaires, c'est en vue des autres qu'il les faut rechercher, en se rendant compte que, sans elles, les objets mêmes qui sont vrais objets de notre étude, sont impossibles à concevoir, à saisir, à recevoir d'aucune façon en partage ${ }^{20}$.

b) La cosmologie d'Aristote est toute différente de celle de Platon. La raison profonde de cette différence est qu'Aristote a toujours refusé, sous une forme ou une autre, la théorie platonicienne des Idées. Sa conception des formes intelligibles immerge celles-ci dans le sensible dont elles constituent l'armature. C'est pourquoi la matière et la forme sont les causes intrinsèques des êtres naturels. Aristote ajoute à ces causes le moteur d'une part, et, à cet égard Aristote salue Anaxagore comme ayant le premier proposé une cause motrice (984 b 15), et la fin de la production, d'autre part, une fin qui, pour les êtres naturels, coïncide avec leur forme. Telle est la théorie des quatre causes, qui peut se réclamer, à certains égards, de Platon, mais qui change profondément le rôle dévolu à la forme intelligible. Or puisque les formes intelligibles sont éternelles, l'Univers l'est également pour Aristote, qui n'a rien à faire d'un Démiurge. Il lui suffit qu'il existe un Premier Moteur, dont il pose l'existence aux livres VII et VIII de la Physique, et dont il précise la nature intellectuelle et immatérielle au livre Lde la Métaphysique.

Selon la métaphysique d'Aristote, qui vient d'être brièvement esquissée, le mouvement exige explication certes, mais son existence $n$ 'a pas besoin d'être prouvée : c'est une évidence que tous les physiologues ont reconnue et contre laquelle sont vains les arguments des Eléates, même si, comme nous le verrons, Aristote s'appliquera à les réfuter. On n'a pas besoin, non plus, comme le faisait Platon, de faire appel à l'âme, que ce soit l'Ame du monde ou les âmes particulières. Puisque la forme est constitutive, avec la matière, des êtres naturels, et que cette forme est également leur fin, il faut dire que c'est la nature ellemême, composée de matière et de forme, qui est chez eux « la cause du mouvement et du repos " (Physique, 192 b 21). Chaque être, par sa nature, tend à la pleine réalisation de sa forme : voilà ce qui définit le mouvement naturel, dont l'opposé est le mouvement violent, celui qu'un agent imprime à un mobile, et dont l'exemple-type est le mouvement mécanique. D'emblée Aristote oppose le dynamisme naturel au mécanisme artificiel. Le second règne dans tous les arts, mais les êtres naturels se distinguent des objets artificiels en ce qu'ils ont en eux-mêmes la cause de leur mouvement. En cela Aristote se distingue nettement de ses prédécesseurs. Ces derniers expliquaient généralement par des mouvements giratoires l'accumulation locale des quatre éléments. Aristote offre sa propre théorie des éléments à partir des qualités premières qui se présentent en deux couples d'opposés : chaud/froid; humide/sec. Ce qui est froid et sec, c'est la terre. Ce qui est froid et humide, c'est l'eau. Ce qui est chaud et humide, c'est l'air. Ce qui est chaud et sec, c'est le feu. La terre et l'eau ont un mouvement naturel vers le bas (de l'Univers); l'air et le feu ont un mouvement naturel vers le haut. Ainsi la disposition des éléments ne tient plus à des causes mécaniques, comme c'était le cas chez les prédécesseurs et partiellement encore chez Platon, mais à leur nature qui tend à son lieu naturel. Au-dessus de ces éléments courent les mouvements circulaires, simples et parfaits, de l'éther, dont sont faites les sphères concentriques, dont le centre est la Terre, et dont les surfaces sont parsemées d'étoiles ou de planètes. Aristote utilise sur ces

${ }^{19}$ ibid., pp.397-398

${ }^{20}$ Timée, 68 e - 69 a ; traduction de J.Moreau un peu modifiée (dans l'édition de la Pléiade) 
mouvements de l'éther, les leçons d'Eudoxe et de Callippe, astronomes de l'Académie, tout en les complétant. Ces astronomes avaient repris l'enseignement des pythagoriciens sur les mouvements réguliers des astres, Pour Aristote le monde supralunaire est tout entier déterminé par ces mouvements réguliers (circulaires et uniformes) auxquels obéissent les étoiles et les planètes disposées sur les sphères et subissant l'attraction des moteurs (au nombre de 55, qui est le nombre des sphères), dont le premier (qui entraîne la sphère des fixes) est le Premier Moteur (Métaphysique,L 8,1073 a 15 - 1074 b 15). Cette extrême régularité du monde supralunaire contraste avec le caractère bigarré du monde infralunaire, beaucoup moins ordonné. Certes, des mouvements naturels y existent encore, comme on l'a vu pour les éléments, et comme il faut le postuler également pour toutes les espèces naturelles, qui sont éternelles, mais l'entrechoc de tous ces mouvements naturels, plus ou moins haut placés dans l'échelle de ces êtres mobiles, est lui-même accidentel, si bien qu'au sein d'un certain règne de la finalité, elle-même imparfaitement coordonnée puisqu'il n'y a ni Démiurge ni Ame du Monde, se produisent des phénomènes contingents, à propos desquels il faut parler de hasard et de nécessité purement matérielle. Platon avait parlé, on l'a vu, de « causes nécessaires « et de «causes divines». Aristote fait appel à une distinction analogue, mais différente, quand, étudiant en biologiste les espèces naturelles, il oppose « la nature nécessaire » et " la nature selon l'essence ». Michel Crubellier et Pierre Pellegrin ont bien montré que l'organisation même des êtres vivants est redevable de cette double causalité dont les effets s'entremêlent d'une façon qui peut nous émerveiller mais également nous étonner : «La règle générale, c'est que la nature fait au mieux avec ce qu'elle a à sa disposition. D'où les formules récurrentes, parfois pittoresques, dont on a déjà parlé - par exemple, celle qui compare la nature à un maître de maison économe -, par lesquelles Aristote décrit cette sorte de négociation entre les exigences de la matière et de ses lois d'un côté et l'action de la finalité de l'autre. Il faut donc déceler le savoir-faire de la nature finalisée. « Disons comment, la nature nécessaire étant donnée, la nature selon l'essence a tiré parti en vue d'une fin de ce qui existe par nécessité » dit Aristote dans les Parties des animaux à propos des cornes (663 b 22). La « nature nécessaire », c'est ce que nous appellerions l'ensemble des lois physiques et chimiques qui gouvernent l'univers. Pour Aristote, cette expression n'est pertinente que dans le monde sublunaire. C'est cette nature seule que reconnaissent les philosophes mécanistes critiqués par Aristote. La « nature selon l'essence », comme on l'a vu plus haut, c'est la nature en tant qu'elle est dirigée vers des buts, à savoir la forme des vivants. Quelquefois Aristote ne peut se retenir d'admirer l'astuce de la nature qui « s'en sort» bien dans un contexte difficile. Dans la Génération des Animaux il conclut: "De sorte qu'encore une fois la nature a astucieusement tiré parti de ce qui est donné »(II,6,745 a 31), dans un contexte qui ne suscitera pas la même admiration chez tout le monde, puisque Aristote trouve remarquable que la nature ait fait coïncider la perte des dents avec la fin de la vie ! Dans le cas des cornes ou des dents, la nature selon l'essence montre son intelligence, par exemple en ne donnant pas de cornes aux animaux très grands comme les éléphants, parce que leur taille suffit à assurer leur sauvegarde ${ }^{2}{ }^{21}$.

Telles sont les grandes lignes de la cosmologie d'Aristote, différente de celle des présocratiques comme de celle de Platon. On a déjà vu que cette nouvelle cosmologie impliquait une nouvelle définition de la nature pour chaque être naturel. Quand on suit le livre de la Physique on s'aperçoit que toutes les notions qui sont fondamentales pour la représentation de la Nature en général se trouvent redéfinies. Après celle de nature, c'est celle de mouvement. L'éléatisme avait exclu le mouvement de l'être et l'avait mis dans la région du non-être. Au plan de l'être intelligible, Platon avait répliqué en proposant un certain non-

${ }^{21}$ M.CRUBELLIER et P. PELLEGRIN, Aristote, le philosophe et les savoirs, Editions du Seuil, 2002,pp.299-300 
être qui est « autre », et, de fait, chez Platon, le mouvement est « autre » que le repos. Au plan de l'être mobile qui est aussi, pour Aristote, intelligible, puisqu'il est gouverné par des "formes », Aristote propose une autre distinction, dont il est le promoteur en philosophie, celle de l'acte et de la puissance. Un être en acte est différent d'un être en puissance, mais ce dernier n'en a pas moins une existence, de même que la matière a une existence par rapport à la forme qui lui procure son unité. Par cette métacatégorie de « puissance », Aristote parvient, le premier, à donner une définition du mouvement : « l'acte de ce qui est en puissance en tant que tel » ou plus exactement « l'entéléchie de ce qui est en puissance, en tel que tel, voilà le mouvement » (201 a 11). Par le terme d' "entéléchie », dont il est l'inventeur, Aristote désigne littéralement l'état de ce qui est en poursuite ou en possession de sa fin, mais en précisant que cet état appartient ici à l'être qui ne possède cette fin qu'en puissance, et en ajoutant que c'est en tant que tel ( en tant que l'être demeure «en puissance » de cette fin) qu'il y a mouvement. Beaucoup de commentateurs parlent d'un "acte inachevé », et ils traduisent sans doute bien l'idée d'Aristote. La difficulté réside en ce qu'Aristote dispose de deux termes pour parler ' " acte ": il y a l'energeia, ou l'acte dans sa forme même, qu'on pourrait traduire par « l'actualisation» et il y a l' entelecheia, ou l'acte dans sa poursuite ou sa possession, qu' on pourrait traduire par « l'actualité »". Parfois d'ailleurs Aristote semble user indifféremment de l'un comme de l'autre. Mais il est manifeste que, lorsqu'il s'agit de définir le mouvement, c'est le deuxième terme qu'il choisit, car il exprime une transition, qui est ce en quoi, en général, consiste le mouvement. De même, quand il s'agit de définir l'âme, dans le traité De l'Ame, Aristote dit qu'elle est substance, au sens de substance formelle. Et il ajoute que la substance formelle est entéléchie (412 a 21). Il remarque alors que l'entéléchie se prend en deux sens : soit comme la science, soit comme l'exercice de la science, et il ajoute : « il est ainsi manifeste que l'âme est une entéléchie comme la science, car le sommeil aussi bien que la veille impliquent la présence de l'âme, la veille étant une chose analogue à l'exercice de la science, et le sommeil, à la possession de la science, sans l'exercice »(412 a 23-26). Il conclut : " l'âme est, en définitive, l'entéléchie première d'un corps naturel ayant la vie en puissance, c 'est-à-dire d'un corps organisé » (412 a 29-30). Ici l'entéléchie première désigne une possession, mais une possession qui n'implique pas l'actualisation des puissances de la vie; l'âme est capable de cette actualisation mais ne l'exerce pas toujours. Ainsi le mouvement vise à atteindre la fin, mais exclut cette fin, car il se transformerait alors en repos. Immédiatement après la définition donnée dans la Physique, Aristote énumère les quatre sortes de mouvement :selon la qualité (altération), selon la quantité (accroissement/diminution), selon la forme (génération/corruption), selon le lieu (transport). Ailleurs il ne mentionne pas le mouvement selon la forme, et pour une excellente raison : il y a substitution d'une forme à une autre et non transition de l'une à l'autre, selon la doctrine aristotélicienne des formes. Il faudrait alors parler seulement de changement (parfois Aristote confond les deux notions de mouvement et de changement, parfois il les distingue) Partout cependant Aristote affirme la primauté du mouvement local, et cela se comprend aisément puisque c'est le mouvement des sphères célestes, dans la cosmologie d'Aristote, qui est l'origine de presque tous les mouvements importants dans le monde sublunaire, et que ce mouvement est le plus parfait, sans commencement ni fin.

Le traité sur le mouvement embrasse quatre livres dans la Physique d'Aristote (livres III,IV,V,VI). Après les commentaires sur la définition du mouvement, Aristote aborde la question de l'infini. Son rejet de l'infini en acte s'oppose à la doctrine tenue par la plupart de ses prédécesseurs. Cependant l'éternité de l'Univers impose que le mouvement des sphères et

\footnotetext{
${ }^{22}$ Sur la difficulté de traduite l' $\varepsilon v \tau \varepsilon \lambda \varepsilon \chi \varepsilon l \alpha$ aristotélicienne et de l'opposer à $l$ ' $E v \varepsilon \rho \gamma \varepsilon l \alpha$, voir L.COULOUB ARITSIS, L'avènement de la science physique, essai sur la physique d'Aristote, Ousia, Bruxelles-Paris, 1980, pp.265-281
} 
le temps qui leur est lié soient infinis. Il s'agit d'un infini en puissance : on peut les prévoir et les remémorer sans leur assigner de limite. C'est comme pour la suite des nombres. Aristote passe ensuite à l'étude du lieu, qui ne peut nous retenir ici. Cette étude est suivie par celle du vide. Pour Aristote, comme pour Platon, il n'y a pas de vide : par quoi ils s'opposent tous deux aux atomistes. Il est clair que l' existence du vide rendrait le mouvement naturel, tel qu'il a été précédemment défini, impossible; par conséquent c'est l'existence du vide qui est impossible pour Aristote. Chemin faisant, Aristote évoque ce que les modernes appelleront le principe d' inertie, qu'il rejette pour la même raison. En abordant la question du temps, Aristote insiste sur le fait que le temps est une propriété du mouvement, qui n'existerait pas sans le mouvement qui en est le substrat. Il y a d'ailleurs un parallélisme entre la grandeur spatiale, le mouvement et le temps. La propriété d'être continu passe de l'un à l'autre. Cette propriété, qu'il développe dans les livres V et VI est très importante pour Aristote. C'est elle qui lui permet d'introduire la divisibilité infinie (en puissance) de l'étendue, du mouvement et du temps. Il s'oppose notamment à la conception de «lignes insécables ». La ligne n'est pas formée de points, ni le mouvement de moments indivisibles, ni le temps d'instants. C'est par ce biais qu'Aristote aborde les arguments de Zénon. Il n'a pas de peine à réfuter l'argument de la Flèche, puisque, dans l'instant, le mobile n'est ni en mouvement ni en repos. L'instant n'a d'existence pour Aristote que pour une pensée qui arrête le mouvement, de même que le point n'a d'existence que pour la pensée qui assigne une limite à une ligne. Il en résulte que le temps n'est pas composé d'instants, bien qu'Aristote concède que le «maintenant» psychique puisse avoir une certaine étendue de durée, comme en témoigne le langage ordinaire. On aurait pu s'attendre à ce qu'Aristote aborde l'argument du Stade aussitôt après sa réfutation de l'argument de la Flèche, ce qui conforterait l'interprétation des modernes qui y voient l'hypothèse d'une indivisibilité dotée d'un minimum de grandeur. Mais ce n'est pas le cas. Aristote ne réfute le Stade, en utilisant tout simplement une conception relative de la vitesse par rapport à des corps immobiles ou mobiles en sens inverse, qu'après avoir exposé et réfuté les trois premiers arguments. Cette disposition de son argumentation confirme l'hypothèse qu'il s'agit bien d'un ordre offert par Zénon lui-même, et non forgé par Aristote. Mais elle ne contredit pas l'interprétation moderne qui reconstitue, on l'a vu, la dialectique entière de Zénon par la succession de l'hypothèse de la continuité divisible et de l'hypothèse de l'indivisibilité. On peut même dire qu'elle en apporte, sinon une preuve, du moins un signe. Car alors qu'Aristote ne met jamais en cause sa réfutation de la Flèche, il est moins satisfait par sa réfutation de la Dichotomie (et de l'Achille à propos duquel il déclare que " c'est le même raisonnement» $(239$ b 18)) . Cette réfutation consiste à dire qu'à la divisibilité infinie de la grandeur spatiale correspond une divisibilité infinie du mouvement et du temps, et donc que, puisque l'infinité est en puissance, c'est dans un temps fini qu'un mouvement fini, quoique infiniment divisible, est exécuté. Certes Aristote trouve que cette réfutation est « suffisante » mais, au livre VIII de la Physique, quand il reprend, une fois de plus, la question de la continuité du mouvement, il admet que la force des arguments de Zénon repose justement sur la rupture de cette continuité (263 a $4-263$ b 8). Si Zénon décompose le mouvement en moitiés successives, il en résulte qu'il arrête à chaque étape le mouvement, et que tout arrêt exige du repos et du temps. Si les arrêts sont infinis, le temps sera infini, et la distance finie ne pourra jamais être parcourue. Sa conclusion est fort claire à cet égard : " A celui qui demande s'il est possible de parcourir l'infini, soit dans le temps, soit dans la longueur, il faut répondre : en un sens oui, en un autre non. Si l'infinité en effet existe en acte, c'est impossible, si c'est en puissance c'est possible. » $(263$ b 2 -5 $) .{ }^{23}$. Quand les modernes reprendront la question dans l'hypothèse d'une infinité en acte, il leur faudra

\footnotetext{
${ }^{23}$ La question est étudiée dans tout son contexte historique et épistémologique par Maurice CAVEING, Zénon et le continu, Paris, Vrin, 1982 et 2002
} 
s'appuyer, dès le XVII ${ }^{\mathrm{e}}$ siß̧cle, sur l'existence de séries convergentes et, plus tard, sur la dissociation entre la composition et la mesure des grandeurs. Aristote ne pouvait prévoir ce développement des mathématiques de l'infini, mais, dans les limites des mathématiques de son temps, sa position était remarquablement cohérente.

\section{5) le stoïcisme et le néo-platonisme}

Après les audaces spéculatives de Platon et d'Aristote, qui répondent à celles de Démocrite, la philosophie de la nature ne témoigne pas, chez les Grecs, d'une même originalité à partir du $\mathrm{III}^{\mathrm{e}}$ siècle avant notre ère. On a vu qu'Epicure s'est contenté de perfectionner, pour y inclure la contingence, l'image du Monde qu'il tenait de Démocrite. Le stoïcisme semble emprunter quelque chose à Platon, puisqu'il fait appel, lui aussi, à l'Ame du Monde, qu'Aristote avait mise de côté au profit de la nature, qui pousse tous les êtres naturels à tendre vers leur fin. Mais l'on verra que le stoïcisme tourne le dos au platonisme et revient à Héraclite. On ne peut en dire autant évidemment du néo-platonisme, qui, au $\mathrm{III}^{\mathrm{e}}$ siècle de notre ère, retrouvera la transcendance platonicienne de l'Intelligence et des Intelligibles, et accordera à l'Ame un rôle de direction dans le sensible. Mais la tâche de l'Ame est plutôt de se tourner vers l'Intelligence, et même, pour les plus favorisés, vers l'Un, qui est la première hypostase, que de faire régner l'ordre dans le monde matériel. Sans se désintéresser du Monde, la philosophie grecque, après Platon et Aristote, semble davantage préoccupée d'éthique que de physique, de mystique que de politique. Les Stoïciens veulent adhérer au Destin, et les néo-platoniciens se tourner vers la contemplation. On ne nie plus le devenir, comme avait eu l'audace de le faire Parménide, mais on se propose soit d'y faire face par l'action raisonnable, soit de s'en détacher par la conversion intérieure. La sagesse prend le pas sur la science, si bien que la philosophie de la nature piétine.

a) Les premiers Stoïciens n'en avaient pas moins une "physique », une discipline qu'ils proposaient à côté de la « logique » et de « l'éthique ». Il est remarquable qu'ils n'admettaient d'aucune façon une " philosophie première » ou une «métaphysique ». Pour eux, l'idée est un « exprimable», dont ils reconnaissaient l'incorporéité, comme pour le lieu, le vide, et le temps. Mais ces incorporels ne sont pas des êtres véritables, ils ne peuvent ni agir ni pâtir, ils appartiennent seulement à notre représentation. L'être est uniquement corporel, mais ce n'est pas l'atome de Démocrite, passif et insensible. C'est une activité ou une force perpétuellement en acte. D'où la différence avec l'aristotélisme : « Le mouvement est à chacun de ses instants un acte et non point un passage à l'acte $»^{24}$. La dynamique stoïcienne ne s'appuie sur aucune force extérieure au corps où elle agit, et pourtant elle peut se diffuser dans l'Univers entier. C'est un aspect de la physique stoïcienne qu'E.Bréhier, il y a un demi-siècle, a bien mis en évidence : "Cette dynamique... ne peut avoir son plein sens que grâce à un dogme physique des plus étranges et des plus indispensables du stoïcisme, celui du mélange total : deux corps peuvent s'unir en se mêlant par juxtaposition, comme on peut mêler des graines d'espèces différentes, ou en se confondant en un, comme dans un alliage de métaux; mais ils peuvent aussi se mélanger d'un mélange total, de façon à s'étendre sans rien perdre de leur substance et de leurs propriétés, l'un à travers l'autre, si bien qu'on trouve à la fois ces deux corps en quelque portion que ce soit de leur espace commun; c'est ainsi que l'encens s'étend à travers l'air, le vin à travers la masse d'eau à laquelle on le mélange, fût-ce celle de la mer entière. Or c'est de cette manière que le corps agent s'étend à travers le patient, la Raison à travers la matière et l'âme à travers le corps. L'action physique ne peut se concevoir que grâce à la

${ }^{24}$ SIMPLICIUS, Commentaire des catégories, 78 b (Arnim,II,n499), cité par E.Bréhier, Histoire de la Philosophie, I, 2,p. 273 
formelle négation de l'impénétrabilité ; c'est l'action d'un corps qui en pénètre un autre et qui est partout présent en lui. C'est ce qui donne au matérialisme stoïcien ce caractère si particulier qui le rapproche du spiritualisme. Le souffle matériel (pneuma) qui traverse la nature pour l'animer est tout prêt à devenir esprit pur ${ }^{25}$. On conçoit alors comment les Stoïciens ont pu reprendre le thème platonicien de l'Ame du Monde : il l'ont matérialisée, en s'inspirant de la cosmobiologie d'origine chaldéenne et de la tradition médicale grecque. J.Moreau, qui a consacré un livre à cet étrange renversement d'un thème qui remonte à Platon et retourne à Héraclite, a pu écrire à cet égard : "L'animisme des Stoïciens, cherchant à se représenter la force qui anime le monde, ne pouvait d'abord trouver mieux que cette représentation physiologique du pneuma. Ayant admis, avec la tradition médicale, que l'âme humaine est un pneuma contenant de la chaleur, que c'est grâce à lui que nous respirons et par lui que nous sommes mus, qu'il est naturellement uni à notre corps et répandu sans solution de continuité dans toute son étendue, qu'il s'exprime dans les fonctions mentales, sensorielles, dans l'élocution et l'activité génésique, ils ont émis l'hypothèse que l'Univers lui-même était également un être vivant, animé, pénétré dans toutes ses parties d'un pneuma qui en assure la cohésion et la permanence $»^{26}$. On ne peut retracer ici la cosmologie qui raconte la transformation du feu primitif et le retour des éléments dispersés à ce même feu ; ce récit nous est si mal parvenu qu'il faut faire confiance à l'imagination pour en restituer les lacunes. Ce qui est sûr, c'est que le retour éternel est régi par un déterminisme implacable, qui est plutôt un fatalisme, et qui n'a rien de commun avec le déterminisme scientifique, tel que nous l'entendons depuis que la physique est devenue mathématique. Sur ce point E.Bréhier avait émis une mise en garde : "Il ne faudrait pas confondre ce destin avec notre déterminisme scientifique. Il n'a rien produit chez les Stoïciens qui ressemble à nos sciences de lois, dont on trouve au contraire l'idée dans les doctrines fort différentes, celles des sceptiques. C'est que la nécessité causale, telle que nous la concevons, est celle d'une relation, et une relation laisse tout à fait indéterminé le nombre des phénomènes qui peuvent s'y soumettre; au contraire le destin de l'univers est comme le destin d'une personne; il s 'applique à un être individuel, l'univers, qui a un commencement et une fin ${ }^{27}$.

Or le destin d'une personne, c'est ce dont s'occupe la morale; il n'y a donc rien d'étonnant à ce que le stoïcisme de l'époque impériale se soit concentré sur l'éthique, en tant que l'éthique doit apprendre à l'homme comment insérer son destin individuel dans le Destin de l'Univers. L'erreur serait de les opposer; la sagesse consiste à les identifier. Jacques Brunswicg, qui s'est appliqué à manifester l'unité du système stoïcien, a pu montrer que « les fondateurs du Stoïcisme auraient pu s'apercevoir (s'ils étaient revenus à l'époque impériale) qu'ils n'étaient pas trahis par leurs successeurs $»{ }^{28}$. Sénèque, Epictète et Marc-Aurèle n'ont rien abandonné, en effet, des principes du système, mais les circonstances assez tragiques de leur vie personnelle, les conduisaient nécessairement à mettre l'accent sur la responsabilité du sage, qui doit épouser son destin et ne pas le subir en esclave. Le seul moyen de parvenir à cet accord, c'est l'appropriation, cette oikeiôsis, qui loin de conduire au repli sur soi-même, ouvre l'être humain à ses devoirs familiaux, civiques, philanthropiques. Sur ce point aussi, J.Brunswicg a relevé l'essentiel de la doctrine : "La notion d'oikeiôsis n'est pas, malgré les apparences, strictement égocentrique. Le vivant, originairement " approprié » à lui-même, l'est aussi aux parties qui le composent; il a le «sentiment» de sa propre constitution. Or, lorsqu'il se reproduit, ce sont des parties de lui-même qui prennent une vie autonome, mais

\footnotetext{
${ }^{25}$ E.BREHIER,op.cit.pp. $274-275$

${ }^{26}$ J.MOREAU, L'Ame du Monde de Platon aux Stö̈ciens,Paris, 1939, reproduit chez Olms, Hildesheim, 1965 , pp.165-166

${ }^{27}$ E.BREHIER, op.cit.p. 279

${ }^{28}$ J.BRUNSWICH, Le Savoir grec, op.cit.,p. 1043
} 
auxquelles il reste attaché, comme le montre déjà le comportement parental chez l'animal. Par ce biais, les Stoïciens pensent pouvoir fonder en nature, par un mouvement d'élargissement progressif dont le schéma survivra à bien des objections et à bien des démentis, l'altruisme familial et civique, et pour finir la philanthropie cosmopolitique, que soutient l'idée d'une justice naturelle au genre humain. A cet égard, il faut au moins signaler d'un mot la greffe du Stoïcisme sur le droit romain, notamment sur l'édifice d'un « droit des gens » international ; c'est un juriste romain, Marcien, qui nous a conservé la définition chrysippienne de la loi (Chrysippe fut le second successeur du fondateur Zénon de Cittium, à la tête de l'Ecole) : " reine de toutes choses divines et humaines », " règle du juste et de l'injuste, qui prescrit aux animaux naturellement politiques ce qu'ils doivent faire, et leur interdit ce qu'il doivent ne pas faire $»^{29}$

b) Le néo-platonisme est très postérieur au stoïcisme, puisqu'il est né, au $\mathrm{III}^{\mathrm{e}}$ siècle de notre ère, dans les cercles platoniciens qui éprouvaient quelque difficulté à prendre le Timée de Platon à la lettre, d'autant plus que Platon lui-même n'avait plaidé, on l'a vu, que pour sa vraisemblance. Ce qui était troublant dans le récit du Timée, c'était la succession des actes du Démiurge, qui faisait dire à certains que cette succession s'opérait dans le temps. En réalité, il ne pouvait s'agir, chez Platon que d'une succession intemporelle, une suite d'actes qui n'a lieu que dans l'éternité. Mais les choses deviennent plus claires, si l'on abandonne toute suite ordonnée: c'est dans l'éternité que la Nature se détache du monde intelligible, par une " émanation » comme on dira plus tard, par une " procession », comme se contente de dire Plotin, le fondateur de l'Ecole, à laquelle le $\mathrm{XIX}^{\mathrm{e}}$ siècle a donné le nom de "néoplatonisme ». Ce néo-platonisme doit beaucoup à Aristote, dont les écrits s'étaient répandus entre temps. Aristote avait proclamé, contre Platon, l'éternité du Monde (ou, si l'on préfère, son caractère sempiternel, qui implique l'infinité du temps). Les néo-platoniciens suivent Aristote sur ce point et s'opposent, par là, aux chrétiens qui s'efforcent, de leur côté, d'élaborer un néo-platonisme chrétien (dont S. Augustin est le plus illustre représentant). Mais les néo-platoniciens doivent encore une autre doctrine à Aristote : c'est l'identité de l'Intelligence et des Intelligibles, qui se trouvait réalisée, pour Aristote, dans l'acte d'intellection. Pour Plotin, l'Intelligence, qui contient les Intelligibles, est la deuxième hypostase (ou sujet d'existence) après l'Un, qu'il identifie au Bien, tel que l'avait conçu Platon dans la République. De l'Un-Bien tout procéde, d'abord L'Intelligence avec les Intelligibles, ensuite l'Ame, qui, en contemplant les Intelligibles, constitue la Nature. Le Démiurge a disparu, mais l'Ame s'est substituée à lui, ce qui éloigne le néo-platonisme de l'aristotélisme, et le rapproche du stoïcisme. Chez Aristote, en effet, comme le constate E.Bréhier dans son Histoire de la Philosophie, "l'âme comme siège de la destinée, a disparu ». Le néo-platonisme va lui rendre l'intégrité de son rôle. "Au contraire, poursuit E.Bréhier,dans le Phèdre, le Timée et les Lois, comme chez les Stoïciens, il y a une âme du monde, rectrice du monde sensible, à laquelle les âmes individuelles, âmes des astres et âmes des hommes, sont consubstantielles et dont elles ne sont que des fragments. Ce n'est pas là une différence de terminologie, mais une opposition profonde dans la conception de l'univers et de la destinée ; de l'univers d'abord qui est un être vivant et dans lequel, par conséquent, les mouvements généraux (mouvements circulaires des astres) sont dus non pas à la propriété d'une quintessence (aristotélicienne) dont la nature est de se mouvoir circulairement, mais à l'influence d'une âme qui domine l'élément igné qui compose le ciel et lui fait prendre, contrairement à sa nature, le mouvement circulaire, ce mouvement de retour sur soi, qui est une imitation du sien propre (Ennéades, II,2;II,1); il n'est rien qui fasse plus horreur au Platonicien que la quintessence aristotélicienne ; partisan de l'unité substantielle du cosmos et

${ }^{29}$ ibid.pp. 1054-1055 
de la sympathie de ses parties, il y voit, non sans raison, la négation de cette thèse. Opposition aussi dans la conception de la destinée, puisque les âmes individuelles ont, dans le détail du gouvernement des choses, le même rôle que l'âme du monde a dans l'ensemble ; leur destinée fait donc partie d'un plan d'ensemble et Plotin développe avec prédilection la vieille image des diatribes, le monde, théatre où la providence assigne à chacun son rôle (Ennéades, III, 2 et $3 » 30$.

Si l'on écarte l'idée de l'éternel retour, il y a donc une certaine parenté entre l'Univers tel qu'il est vu par le Stoïcien et l'Univers tel qu'il est vu par le Néo-Platonicien. Pourtant une grande différence se fait jour, qui est la différence entre cette sorte de matérialisme d'une part et cette sorte de spiritualisme d'autre part. Pour les Stoïciens le fonds matériel n'est pas mauvais, puisque tout vient du feu purificateur et tout y retourne : rien n'échappe à la puissance du Souffle divin. Pour le néo-platonisme, il y a quelque chose qui échappe à la puissance de l'Ame du Monde et des âmes individuelles : c'est la matière, dans laquelle ne s'inscrit que passagèrement la volonté de l'âme, car la matière n'est pas seulement indéterminée, comme chez Aristote, elle est indéterminable, rebelle à toute inscription profonde du monde intelligible. Telle est l'origine du mal. Comme l'explique E.Bréhier : « Le mal n'est pas une simple imperfection puisque, alors, il faudrait dire que l'Intelligence est mauvaise parce qu'elle est inférieure à l'Un. Vice, faiblesse de l'âme, tout ce qui paraît être le mal en soi, n'est mal que parce que l'âme est entrée en contact avec la matière, est plongée dans le devenir à cause de ce contact; elle s'en purifie non pas en s'en rendant maîtresse, mais en la fuyant. Si cette matière existe pourtant, c'est parce qu'il faut que tout degré de réalité soit épuisé ; elle n'est pas indépendante de l'Un; elle en est seulement comme le dernier reflet, avant l'obscurité complète du néant (Ennéades, I,8) ${ }^{31}$. Bien que cette métaphysique néo-platonicienne ait subi des retouches, puisqu'elle ait passé successivement entre les mains de Porphyre, Jamblique, Syrianus, Proclus et Damascius, on ne peut pas dire qu'elle ait jamais manifesté une grande confiance dans la destinée de l'âme hors cette fuite de la matière que lui recommandait Plotin. Pour ce dernier, la descente de l'âme consistait dans une défaillance de sa conversion vers l'Intelligence, grâce à laquelle elle était capable de gouverner son corps, et par son attachement corrélatif à son reflet dans un corps qui l'en rendait captive ; ainsi Narcisse, selon le mythe que reprenait Plotin (Ennéades, IV, 3,8,9), se laissait attirer par son image dans la fontaine et s'y noyait. Mais les derniers représentants du néo-platonisme ont cherché refuge dans la magie et dans la théurgie, ce qui était déjà une possibilité offerte chez Plotin lui-même. Si bien qu'une indécision demeure dans le néoplatonisme, s'il s'agit de la sagesse face au devenir : soit qu'il tende, comme Platon lui-même, à s'évader le plus possible du sensible pour réintégrer progressivement l'empyrée d'avant la chute des âmes, soit qu'il s'accommode, comme le fait le stoïcisme, du cours du monde éclairé par les oracles et la divination. On ne sait plus très bien chez les représentants du néoplatonisme, qui s'opposent au christianisme dans les premiers siècles de notre ère, si la religion païenne et les cultes traditionnels, qu'ils défendent âprement, élèvent l'âme vers le Ciel ou la rivent à la Terre. Les sentiments religieux peuvent avoir des effets contraires, qui sont manifestes dans le néo-platonisme.

\section{Conclusion}

Si la reconstruction qui vient d'être proposée des doctrines philosophiques du mouvement dans l'antiquité gréco-romaine, si schématique qu'elle soit, est exacte, alors on comprend

\footnotetext{
${ }^{30}$ E.Bréhier, op.cit., p.405

${ }^{31}$ ibid., p. 408
} 
assez bien que la philosophie d'Aristote, assez fidèle à l'expérience commune, ait régné au Moyen-Age et que, pour s'en défaire à partir de la Renaissance, on ait invoqué le patronage de Platon. Au temps des ingénieurs et des artistes, qui bâtissaient des villes et ornaient leurs palais, la figure du Démiurge, qui ordonne le monde dans de justes proportions, paraissait certainement plus attirante qu'une nature diversifiée, accueillante à l'observation, mais résistante à l'expérimentation, telle que celle qu'avait proposée Aristote. Quand Galilée invente les principes d'inertie et de relativité, qui n'étaient pas admissibles dans la perspective aristotélicienne, il peut s'imaginer retrouver les idées du Démiurge, qui soumet le réceptacle du milieu spatial à des lois mathématiques inscrites dans l'Ame du Monde. Il n'était même pas nécessaire de retenir cette hypostase de l'Ame du Monde, que le paganisme avait finalement entourée de divination et de magie. Mais le privilège du mouvement circulaire et uniforme, que Platon, après Pythagore, avait instauré dans l'astronomie était tel que Galilée y lia le principe d'inertie et refusa les lois de Képler qui rompaient avec la tradition des mouvements circulaires et uniformes. Descartes fut, sur ce point, plus novateur que Galilée en liant le principe d'inertie au mouvement rectiligne et uniforme. Plus tard, la distinction des qualités premières et des qualités secondes, empruntée à l'atomisme, devait doter le mécanisme de la physique moderne de principes très puissants qui ont permis les progrès considérables de la science moderne.

A partir du $\mathrm{XIX}^{\mathrm{e}}$ siècle cependant, on se rendit compte que le mécanisme n'avait pas réponse à tout, que la chimie a ses propres lois, sans parler de la biologie, terriblement rebelle. $\mathrm{Ce}$ n'est pas un hasard si le philosophe Schelling, suivi par des physiciens initiateurs de l'électromagnétisme, ressuscita l'idée de l'Ame du Monde, et instaura le concept d'une philosophie de la nature distincte de la science elle-même. On ne peut pas dire que ce concept se soit imposé depuis deux siècles; néanmoins il apparaît indispensable en un temps où la science ne cesse de se diversifier et où la physique elle-même a perdu son unité. La curieuse aventure de la science moderne invite, de cette façon, à porter un regard attentif sur son modèle grec, auquel elle peut encore peut-être emprunter quelque chose

Ce que les anciens Grecs peuvent lui apprendre, en effet, avec la naïveté qui entoure leur génie, c'est qu'il y a un ou plusieurs Cosmos, mais que, de toute façon, dans un Cosmos les lois sont les mêmes partout, à moins qu' on ait de bonnes raisons d'y distinguer des régions plus ou moins autonomes. Les Milésiens ont développé cette profonde intuition, qui a été reprise par les Pythagoriciens et ceux qui s'en sont inspirés chez les présocratiques, sans compter Platon et Aristote qui en ont fait, chacun à sa manière, la base de leur cosmologie.

Or précisément, du fait d'un ensemble de circonstances remarquables, la culture scientifique a retrouvé, depuis trois quarts de siècle, l'idée d'une cosmologie. De plus notre cosmologie impose un seul Univers (du moins celui que nous pouvons dire nôtre) qui est fini et qui a un commencement dans le temps. Il faut imaginer, à partir d'une origine commune, où toutes les forces se confondaient, la séparation des types d'interaction, l'activité des étoiles, la constitution de systèmes planétaires, où la vie a, comme sur la Terre, pu naître. Ce sont des problèmes que la science classique n'avait pas abordés (et avec raison sans doute) mais où les penseurs grecs nous avaient précédés. On voit bien aujourd'hui l'impossibilité des solutions qu'ils proposent, et l'on peut s'instruire sur la manière dont ils les ont abordés. Savons-nous éviter les mêmes impasses ? Avons-nous trouvé le moyen sûr de les écarter? Les Grecs peuvent nous instruire par leurs erreurs autant que par les profondes intuitions qui les guidaient. Ils peuvent également nous apporter le témoignage de sagesses qui, par delà les limites de la science, apportent des raisons de vivre. Car, la science et la sagesse ne peuvent coïncider, comme l'éléatisme a essayé d'en apporter la preuve, mais il faut essayer de les conjoindre sans contradiction: toutes les doctrines qu'on a passées en revue, même si certaines luttent difficilement contre l'objection de cette contradiction, se sont efforcées d'en 
triompher. Il est frappant d'ailleurs qu'elles aient pu se rejoindre dans la pratique, comme ${ }^{32}$ c'est le cas de l'épicurisme et du stoïcisme impérial, alors même que leurs théories sont profondément différentes. C'est encore une leçon qu'on peut retirer de la fréquentation des auteurs grecs anciens : il n'est pas nécessaire de s'entendre sur tout pour se retrouver d'accord dans la pratique : la raison ne cesse pas de rapprocher les hommes, même quand elle semble les autoriser à choisir des systèmes de pensée très éloignés les uns des autres.

Hervé Barreau

CNRS Strasbourg-Nancy, UMR 7117, Archives Henri Poincaré, Nancy 2

\section{Bibliographie}

Barnes J.,Schofield M.,Sorabji R. (dir.), Articles on Aristotle, vol.III, Metaphysics, Londres, Duckworth, 1979

Barreau H, Aristote et l'analyse du savoir, Paris, Seghers, 1972

Zénon d'Elée, in Encyclopaedia Universalis, Dictionnaire des Philosophes, Albin Michel, 1998, 1597-1601

La Physique du continu chez Aristote, sa réponse à Zénon, in Le Labyrinthe du Continu, J.M.Salanskis et H.Sinaceur (Eds), Springer Verlag, 1992, 3-15

Brehier E., Histoire de la Philosophie, , T.I, 2 Période hellénistique et romaine, PUF, 8 è édit. 1967

Brisson L., Le même et l'autre dans la structure ontologique du Timée de Platon, Paris,

Klincksieck, 1974,

Brunswicg J., Lloyd G et Pellegrin P.(dir.), Le Savoir grec, Flammarion, 1996

Caveing M., Zénon et le continu, Paris Vrin, 1982 et 2002

Couloubaritsis L., L'Avènement de la science Physique, essai sur la Physique d'Aristote, Paris-Bruxelles, Ousia, 1980

Crubellier M., Pellegrin P., Aristote, le philosophe et les savoirs, Paris, Seuil, 2002

Dumont J.P.,Delattre D.,Poirier J.L., Les Présocratiques, Gallimard, édit.de la Pléiade, 1988

Frère J., Temps, désir et vouloir en Grèce ancienne, Paris-Athènes, Ed.Dioné, 1995

Moreau J., L'Ame du Monde de Platon aux Stö̈ciens, Hildesheim, Olms, 1965

Robin L., La pensée grecque et les origines de l'esprit scientifique,Paris, Albin Michel, 1963 et 1973 